\title{
A SPITZER/IRS SPECTRAL STUDY OF A SAMPLE OF GALACTIC CARBON-RICH PROTO-PLANETARY NEBULAE
}

\author{
Yong Zhang and Sun Kwok \\ Department of Physics, University of Hong Kong, Pokfulam Rd., Hong Kong \\ zhangy96@hku.hk; sunkwok@hku.hk \\ and \\ Bruce J. Hrivnak \\ Department of Physics \& Astronomy, Valparaiso University, Valparaiso, IN 46383, U.S.A. \\ bruce.hrivnak@valpo.edu
}

\begin{abstract}
Recent infrared spectroscopic observations have shown that proto-planetary nebulae (PPNs) are sites of active synthesis of organic compounds in the late stages of stellar evolution. This paper presents a study of Spitzer/IRS spectra for a sample of carbon-rich PPNs, all except one of which show the unidentified $21 \mu \mathrm{m}$ emission feature. The strengths of the aromatic infrared band (AIB), $21 \mu \mathrm{m}$, and $30 \mu \mathrm{m}$ features are obtained by decomposition of the spectra. The observed variations in the strengths and peak wavelengths of the features support the model that the newly synthesized organic compounds gradually change from aliphatic to aromatic characteristics as stars evolve from PPNs to planetary nebulae.
\end{abstract}

Subject headings: infrared: stars — stars: AGB and post-AGB — stars: circumstellar matter

\section{Introduction}

Proto-planetary nebulae $(\mathrm{PPNs})$ represent a relatively short stage $\left(\sim 10^{3} \mathrm{yr}\right)$ when a low- or intermediate-mass star evolves from the asymptotic giant branch (AGB) into the planetary nebula (PN) phase (Kwok 1993; Van Winckel 2003). PPNs have central stars with $\mathrm{B}-\mathrm{G}$ spectral type and are not yet hot enough to ionize the surrounding AGB remnant 
envelope. As descendants of AGB stars, PPNs are expected to inherit many of the observed properties of AGB stars, including infrared excess and molecule-line emission from the dust and molecular components of the circumstellar envelopes of AGB stars. By comparing the spectroscopic properties of the dust component from AGB stars to PPNs to PNs, one sees evidence for a continuous evolution as the result of chemical synthesis and a hardening radiation field (Kwok 2004). While the infrared spectra of AGB stars are characterized by silicates or silicon carbide emission features (Kwok, Volk, \& Bidelman 1997), the aromatic infrared bands (AIBs) dominate the infrared spectra of carbon-rich PNs. How this chemical transition occurs is one of the most interesting question in astrochemistry. PPNs, being in the evolutionary stage between these two phases, offer important clues to the chemical synthesis of organic compounds in the late stages of stellar evolution.

Specifically, while carbon-rich PNs show strong emission features at 3.3, 6.2, 7.7-7.9, 8.6, 11.3, and $12.7 \mu \mathrm{m}$ due to aromatic compounds, these features are not seen in AGB stars and they only begin to emerge during the PPN phase. Furthermore, PPNs show aliphatic features at 3.4 and $6.9 \mu \mathrm{m}$ which are generally weak or absent in PNs (Geballe et al. 1992; Kwok, Volk, \& Hrivnak 1999; Kwok, Volk, \& Bernath 2001). Whether this aliphaticaromatic transition is the result of chemical synthesis or radiative interactions is yet to be determined. In a comprehensive study of the peak wavelengths of the AIB features, Sloan et al. (2007) found that the peak wavelengths of the 7.7 and $11.3 \mu \mathrm{m}$ AIB features are correlated with the temperatures of the central stars, which they interpret to be the result of change in the aliphatic to aromatic content ratio.

The AIB features, although commonly assumed in astronomical literature to be due to polycyclic aromatic hydrocarbon (PAH) molecules, are in fact more likely (at least in the circumstellar environment of late-type stars) to arise from complex, amorphous, impure organic solid-state compounds (Kwok 2009). Examples of such amorphous compounds include hydrogenated amorphous carbon (HAC; Jones, Duley, \& Williams 1990; Scott, Duley \& Jahani 1997), carbon nanoparticles ( $\mathrm{Hu} \&$ Dulev 2008), quenched carbonaceous composites (Sakata et al. 1983), coal or kerogen (Papoular 2001). Common properties among these candidates are a mixed $s p^{2} / s p^{3}$ chemistry, and in the case of coal or kerogen, the presence of elements such as $\mathrm{O}, \mathrm{S}, \mathrm{N}$, in addition to $\mathrm{C}$ and $\mathrm{H}$. A model of such compounds has been presented by Pendleton \& Allamandola (2002).

To complicate the issues further, it is now known that the infrared spectra of Crich PPNs contain two unidentified broad emission features at around 21 and $30 \mu \mathrm{m}$ (see Hrivnak et al. 2009, and references therein). The $21 \mu \mathrm{m}$ feature was first discovered in spectra taken by the Infrared Astronomical Satellite (IRAS) Low Resolution Spectrometer (LRS) (Kwok et al. 1989). Based on an Infrared Space Observatory (ISO) study of eight $21 \mu \mathrm{m}$ 
sources, Volk et al. (1999) found that they all have the same intrinsic profile and peak wavelength $(20.1 \mu \mathrm{m})$. To date, the number of known $21 \mu \mathrm{m}$ sources is very small, 16 in the Galaxy (Hrivnak et al. 2008) and 8 in the Large and Small Magallenic Clouds (Volk et al. 2010). The carrier of the $21 \mu \mathrm{m}$ feature remains unknown. A number of candidates, including TiC, AIBs, fullerenes, hydrogenated amorphous carbon (HAC), nanodiamonds etc, have been proposed (see Speck \& Hofmeister (2004) and Posch et al. (2004) and references therein). The fact that all or almost all the $21 \mu \mathrm{m}$ sources are C-rich PPNs suggests that the carrier of this feature is carbon-based and is associated with a very rapid chemical processing.

The very broad $30 \mu \mathrm{m}$ feature, usually extending from 25 to $45 \mu \mathrm{m}$, was first discovered by Forrest et al. (1981) in C-rich AGB stars and PNs, and subsequently has been commonly detected in C-rich PPNs (Volk et al. 2002). An ISO study of a large sample of evolved stars shows large variations in the peak wavelength and width of the $30 \mu \mathrm{m}$ feature among different sources (Hony et al. 2002). On the basis of the ISO spectra of PPNs, Volk et al. (2002) found that the feature can be resolved into a broad component at $33 \mu \mathrm{m}$ and a narrower component at $26 \mu \mathrm{m}$, although Sloan et al. (2003) argued that the $26 \mu \mathrm{m}$ feature is an artifact caused by an inaccurate calibration. The origin of the $30 \mu \mathrm{m}$ feature is still under debate. It was first suggested as solid magnesium sulfide (MgS; Goebel \& Moseley 1985). Hony et al. (2002) modeled the profile using MgS dust grains with a temperature different from the bulk of dust that contributes to the continuum. They argued that the $26 \mu \mathrm{m}$ feature can be explained by MgS with a different shape distribution. However, Zhang et al. (2009) found that the available MgS dust mass in the circumstellar envelope is too low to account for the observed feature strength. Moreover, the fact that the feature is only detected in C-rich sources and takes up a significant fraction $(\sim 20 \%)$ of the total luminosity of the object seems to favor the more abundant carbonaceous material as possible carrier.

The infrared spectra of C-rich PPNs therefore consist of contributions from several components: a base continuum commonly assumed to be due to thermal emission from amorphous carbon grains, a component due to aromatic and aliphatic compounds, and broad emission features at 21 and $30 \mu \mathrm{m}$. With the Spitzer spectroscopic survey of PPNs, we now have a large enough sample to derive the systematic behavior of the spectral features and draw conclusions on their possible evolution during the transition from the AGB to the PN phase.

In this study, we present Spitzer spectra of a sample of carbon-rich PPNs, with the goal to investigate the spectral behavior of the AIB, $21 \mu \mathrm{m}$, and $30 \mu \mathrm{m}$ features. In order to derive the intrinsic strengths and profiles of the broad and often overlapping emission features, we need to correctly subtract the continuum under the features. A procedure of optimized fitting is used to decompose the observed spectra into individual emission components. The 
paper is organized as follows. In Section 2, we briefly describe the data used in the current study. The method for spectral decomposition is presented in Section 3. In Section 4, we report the measurement results and discuss the implications of our findings. The last section summarizes the conclusions.

\section{Data}

The sample includes ten PPNs, all of which except one (IRAS 01005+7910), are known $21 \mu \mathrm{m}$ sources. The observations were carried out with the Infrared Spectrograph (IRS; Houck et al. 2004) on the Spitzer Space Telescope (Spitzer; Werner et al. 2004) at different epochs between 2004-2008. Most of the data were obtained from the Spitzer archive as part of the program No. 20208 (PI: B. Hrivnak) and 93 (PI: D. Cruikshank). These sources were observed with the Short-High (SH) and Long-High (LH) modules, covering the wavelength range from $9.9-19.6 \mu \mathrm{m}$ and $18.7-37.2 \mu \mathrm{m}$, respectively. The spectra of IRAS $07134+1005$ and IRAS $20000+3239$ (with extremely strong $21 \mu \mathrm{m}$ emission) are not included in our analysis because the $21-\mu \mathrm{m}$ feature is saturated in the observations. We also include the data of three targets (IRAS 01005+7910, IRAS 04296+3429, and IRAS 22223+4327) observed with the short-Low (SL) module in Spitzer programs 30036 (PI: G. Fazio) and 45 (PI: T. Roellig), which cover a larger wavelength range $(5.5-37.2 \mu \mathrm{m})$. These allow the study of the shorter wavelength $6-10 \mu \mathrm{m}$ AIB features. In some cases there are discontinuities in the fluxes between the different modules. This is due to different slit sizes and slight mis-pointing. To ensure that all spectra were on the same flux scale, the regions of spectral overlap are compared and used to scale and combine the spectra derived with different modules for each object. In the case of IRAS $19441+2401$, the star is outside of the SH aperture so the spectrum would only have recorded part of the extended emission. Consequently, the shortwavelength part of the spectrum may not be reliable. Details of the data processing are the same as described in Hrivnak et al. (2009). The extracted spectra are displayed in Figure 1.

\section{Model fitting}

The IRS spectra of all ten sources show strong infrared excess due to dust continuum emission. Superimposed on the continuum are several strong, broad emission features. There is no evidence of strong atomic emission lines as one would expect in a PN. The only narrow

features present are molecular lines. This is consistent with the fact that the central stars of these objects are not hot enough to photoionize the surrounding circumstellar materials. In this section, we report on our efforts to fit the observed spectra with a combination of dust 
continuum, dust features, and $\mathrm{H}_{2}$ lines.

For the spectral decomposition, we used the IDL package PAHFIT originally developed by Smith et al. (2007) to fit the Spitzer IRS spectra of nearby galaxies. The model spectra take into account the contributions from stellar continuum, thermal dust continuum, $\mathrm{H}_{2}$ lines, and AIBs. We modified this code to include fittings to the 21 and $30 \mu \mathrm{m}$ features. The optimal fitting to the observed spectra is achieved through the Levenberg-Marquardt least-squares algorithm. The fitting results are shown with the spectra in Figure 1 .

In our model, the stellar continuum is assumed to be a $5000 \mathrm{~K}$ blackbody for all the sources. While the individual stellar temperatures are known to be mostly in the range of 5250-7250 K, the actual stellar contribution to the mid-infrared spectra is minimal (see Fig. 1). In almost all cases, the effects of photospheric continuum contributions are negligible.

The dominant contribution to the mid-IR continuum is due to dust thermal emission. A modified blackbody model $\left[I_{\lambda} \sim \lambda^{-2} B_{\lambda}(T)\right.$, where $B_{\lambda}(T)$ is the blackbody function with a temperature $T]$ is adopted to simulate the dust thermal continuum. However, for most of the sources, we must use two components with different temperatures to get the best fit. The warm dust component has temperatures from 140 to $180 \mathrm{~K}$, and the cold component has temperatures from 60 to $80 \mathrm{~K}$. The only exception is IRAS 19477+2401, which does not need a warm component (although we note that the short-wavelength spectrum of this object may not be reliable).

Eight $\mathrm{H}_{2}$ rotational lines from $\mathrm{S}(0)-\mathrm{S}(7)$ are included in the fitting. The line profiles are assumed to be Gaussian. The $\mathrm{H}_{2} 0-0 \mathrm{~S}(0)$ para ground-state transition at $28 \mu \mathrm{m}$ is clearly detected in IRAS $04296+3429$ and IRAS $22223+4327$. From the spectra, we can see that the $\mathrm{H}_{2}$ lines are much narrower than dust features.

Also included in the fitting are all the AIB features in Smith et al. (2007, see their Table 3). Drude profiles $\left[I_{\lambda} \sim \frac{\gamma^{2}}{\left(\lambda / \lambda_{0}-\lambda_{0} / \lambda\right)^{2}+\gamma^{2}}\right.$, where $\lambda_{0}$ is the central wavelength, and $\gamma$ is the fractional FWHM] are assumed for each feature. Figure 1 shows that the 7.7, 11.3, and $12.7 \mu \mathrm{m}$ complexes can be well fitted with blended subfeatures. One should bear in mind that these subfeatures do not necessarily represent distinct physical emission bands.

The intrinsic shape of the $21 \mu \mathrm{m}$ feature has been investigated by Volk et al. (1999), who found that the profiles are similar among different sources, all showing "a sharp rise on the short-wavelength side, beginning at $18.5 \mu \mathrm{m}$ to a peak at $20.1 \mu \mathrm{m}$, and a gradual decline on the long-wavelength side to $24 \mu \mathrm{m}$ ". Hrivnak et al. (2009) found the same shape in the Spitzer spectra they studied and we see similar shapes for the three additional objects (IRAS 01005+7910, 04296+4329, 22223+4327) in our sample (see Figure 1). To represent this profile asymmetry, we have assumed an asymmetrical Drude profile with the $\gamma$ value in 
the long-wavelength side three times larger than that in the short-wavelength side.

The very broad $30 \mu \mathrm{m}$ feature is obviously present in the Spitzer IRS spectra. Hony et al. (2002) found that the peak wavelength of this feature shifts from $26 \mu \mathrm{m}$ in some AGB stars to $38 \mu \mathrm{m}$ in PNs. Through the fitting, we find it difficult to reproduce the observations using a single component because the decomposed profile of this feature differ from star to star. Although the Spitzer spectra do not show the two distinct components in the $30 \mu \mathrm{m}$ feature previously deduced from the $I S O$ spectra, we find it practical to use two Drude profiles peaking at $26 \mu \mathrm{m}$ and $30 \mu \mathrm{m}$ to represent this feature as they provide better fits to the data.

Dust extinction is not considered in the present study as its effect is expected to be small in mid-infrared wavelengths. If present, extinction may reduce the emission flux at $9.7 \mu \mathrm{m}$ and $18 \mu \mathrm{m}$ due to interstellar silicate absorption. In some cases, our model cannot reproduce the fluxes around $9.7 \mu \mathrm{m}$ and $18 \mu \mathrm{m}$ (the model fluxes at these wavelengths are slightly higher than observed), which might be an indication of the presence of interstellar dust extinction. Nevertheless, the small discrepancy does not affect our study of dust features.

Although this study is mainly focused on individual features, we explore the possibility of using principal component analysis (PCA) to study global spectral properties and classify PPNs. Our results are presented in Appendix A.

\section{Results and discussion}

We are able to obtain reasonably good fits to the observed spectra using the above model and the results are shown in Figure 1. In Table 1 we give the integrated fluxes of the continuum and the 21 and $30 \mu \mathrm{m}$ features. The flux fractions of the 21 and $30 \mu \mathrm{m}$ features to the total IR spectra are also presented in this table. Table 2 lists the strengths of all the major AIB features. Since the SL observations were made only for three objects (IRAS 01005+7910, IRAS 04296+3429, and IRAS 22223+4327), we are only able to obtain the $6.2,6.9,7.4,7.8,8.3$, and $8.6 \mu \mathrm{m}$ fluxes for these three objects but not the others. The feature strengths are derived by integrating the fluxes above the model (star + dust) continuum. To extract the strengths of dust features, some authors (e.g., Bernard-Salas et al. 2009) derived the continuum through fitting a spline function to selected spectral regions free from feature emission. From the PPN spectra shown in Figure 1, we can see that the features are broad and are blended with each other. Consequently, it is difficult to find a region completely free of dust features to adequately define the baseline. Given the difficulty in selecting appropriate sections of the spectra to represent the continuum, we believe that our present method gives more robust results. 
For almost all of the targets, two dust components with different temperatures are required to reproduce the thermal continuum. The blackbody temperatures used for the fitting are listed in Table 1. The cooler component probably arises from an extended circumstellar envelope left over from the AGB progenitors, and the warm component may originate from a central torus or more recent mass loss. Some PPNs have been found to exhibit equatorial tori. For example, the dust continuum and CO map of the $21 \mu \mathrm{m}$ source IRAS $07134+1005$ suggests the presence of torus (Kwok, Volk, \& Hrivnak 2002; Meixner et al. 2004; Nakashima et al. 2009).

Tables 1 and Table 2 also give the fitting uncertainties of the feature strengths, which are estimated using the full covariance matrix of the least-squares parameters. We can see that the errors are typically less than 20\%. Adding more blackbody components with temperature of 50-200 $\mathrm{K}$ to the fitting might lead to $5-15 \%$ changes in the fluxes of the broad $30 \mu \mathrm{m}$ feature, but will have minimal effect on those of the other features $(<5 \%)$.

\section{1. $\quad 21 \mu \mathrm{m}$ and $30 \mu \mathrm{m}$ features}

The strengths of the $21 \mu \mathrm{m}$ feature vary among the sources. In the strongest $21 \mu \mathrm{m}$ source (IRAS 06530-0213) in our sample, the fraction of the flux emitted by the $21 \mu \mathrm{m}$ feature to the total emitted infrared flux amounts to $\sim 5 \%$. Since we fixed the peak wavelength and shape of the $21 \mu \mathrm{m}$ feature in the fitting process, the good agreement between the model and observation supports the suggestion that the intrinsic profile of the $21-\mu \mathrm{m}$ feature does not change in different sources (Volk et al. 1999). We also note that the two PPNs (IRAS $19477+2401$ and IRAS 22223+4327) with weak $21 \mu \mathrm{m}$ features have no or weak warm dust component. This might indicate that the $21 \mu \mathrm{m}$ feature mostly arises from the warmer torus, although infrared imaging is needed to confirm this suggestion. The one object in our sample without the $21 \mu \mathrm{m}$ feature is IRAS $01005+7910$. It has a stellar temperature of $21000 \mathrm{~K}$, which is considerably hotter than the other PPNs (see Table 1). The presence of the $21 \mu \mathrm{m}$ feature in PPNs but not in AGB stars or PNs has previously been interpreted as an indication of the evolution of the feature due to chemical synthesis or changing excitation conditions.

The $30 \mu \mathrm{m}$ feature is present in all of the PPNs. Its flux typically accounts for $\sim 20 \%$ of the total infrared flux in the PPNs (they range from 8 to 28\%). From Figure 1 one can see that the $30 \mu \mathrm{m}$ feature has an asymmetric profile with a sharp blue wing and a broad red tail. Although the presence of two separate components for this feature as seen in the ISO spectra has been shown to be unreal (Hrivnak et al. 2009), we find it helpful to use two components to fit this broad feature because a single component of fixed shape and central 
wavelength cannot give a satisfactory fitting. We should note that the two components do not necessarily mean two individual features. We find that the relative flux ratios of the two components vary among sources. This representation of the changing shape and peak is consistent with the findings of Hony et al. (2002) who simulated the MgS emission feature and found that the peak position changes with the grain shape and temperature.

Bernard-Salas et al. (2009) investigated the Spitzer/IRS spectra of 25 PNs in the Magellanic Clouds (MCs) and found that about half of them possess the $30 \mu \mathrm{m}$ feature. They did not find a clear relation between the presence of this feature and nebular sulfur abundance, which is used to argue against the suggestion of $\mathrm{MgS}$ as possible carrier. The strengths of the $30 \mu \mathrm{m}$ features in MC PNs (see Figure 6 in Bernard-Salas et al. 2009) are similar to those in MW PNs, suggesting that the different metallicity of the two galactic systems is not a major factor in the existence of the $30 \mu \mathrm{m}$ feature. However, when comparison is made to the strengths of the feature in MW PPNs as presented in this study, one sees that the feature is stronger in PPNs. This suggests that the $30 \mu \mathrm{m}$ carrier is gradually being destroyed or not excited in the PN phase. If MgS is the carrier, we expect the amount of sulfur released into the gaseous nebula will increase as the star evolve to a PN. However, the low sulfur abundance found in PNs (e.g. Pottasch \& Bernard-Salas 2006; Bernard-Salas et al. 2008) does not seem to support this idea (although there exist other explanations for the 'sulfur anomaly'; see Henry et al. 2006). The decline of the strength of the $30 \mu \mathrm{m}$ feature is more likely to be the result of changing physical or chemical conditions.

\subsection{AIBs}

The AIB features are prominently present in all of the PPNs in this sample and the peak wavelengths of the features detected are given in Table 3 . The profiles and central wavelengths of AIBs are known to vary in different astronomical sources (see, e.g. Peeters et al. 2002). Almost all of the sources have the $11.3 \mu \mathrm{m}$ feature peaking at the same wavelength, $11.39 \pm 0.03 \mu \mathrm{m}$. The exceptions are IRAS $22574+6606$ and IRAS $01005+7910$, the latter of which is a much hotter source and does not possess the $21 \mu \mathrm{m}$ feature.

In order to examine the features in detail, we have plotted in Figure 2 the continuumsubtracted spectra of the three PPNs observed in the IRS mode SL 6-10 $\mu \mathrm{m}$ region together with the spectra of the young PN IRAS 21282+5050. The peak wavelengths of the aromatic and aliphatic emission features are marked. The $6.2 \mu \mathrm{m}$ aromatic feature is detected in all three PPNs that were observed in this wavelength region. The 6.9 and $7.25 \mu \mathrm{m}$ aliphatic features are particularly distinct in IRAS $04296+3429$, whereas the $8.6 \mu \mathrm{m}$ aromatic feature is relatively weak in that object. In IRAS $22223+4327$, the $6.9 \mu \mathrm{m}$ is not obvious from 
looking at the spectrum alone, but from the fitting results, the integrated strength of this feature is quite high due to its width.

One can compare the $6.2 \mu \mathrm{m} / 11.3 \mu \mathrm{m}$ intensity ratio to that found in PNs in the $\mathrm{MW}$, LMC, and SMC (Bernard-Salas et al. 2009, see their Fig. 2). For IRAS 04296+3429 and IRAS $22223+4327$, the ratio is low, 0.45 and 0.47 , respectively, while for the hotter PPN IRAS $01005+7910$, the ratio of 1.40 is similar to that found for most of the PNs. An examination of the shorter wavelength features also shows shifts in the peaks of the features between IRAS $01005+7910$ and the other two sources observed and also the appearance of a small shift in the peak of the broad 7.7-8.2 $\mu \mathrm{m}$ complex between IRAS $22223+4327$ and $04296+3429$. Also seen is a difference in the peak at $7.60 \mu \mathrm{m}$ for the hot PPN IRAS $01005+7910$ and $7.85 \mu \mathrm{m}$ for the PN IRAS 21282+5050. There seems to be a general trend for the peak wavelength of the $7.7 \mu \mathrm{m}$ feature to shift to shorter wavelengths with evolution.

In Figure 3, we have plotted the peak wavelengths of the $11.3 \mu \mathrm{m}$ feature as functions of the fractional strengths of the of the $21 \mu \mathrm{m}$ feature. The only two objects with redshifted $11.3 \mu \mathrm{m}$ are the ones with weak or no $21 \mu \mathrm{m}$ feature. The peak wavelengths of the $11.3 \mu \mathrm{m}$ feature as a function of central star temperatures are plotted in Figure 4. For comparison, we have also included in Figure 4 the values for Ae/Be and other C-rich stars from Sloan et al. (2007) and Keller et al. (2008). Although these objects do not belong to a homogeneous sample, this figure seems to suggest that the peak of the $11.3 \mu \mathrm{m}$ feature occurs at shorter wavelengths at higher central-star temperatures. It has been suggested that the sources with most redshifted AIB features are exposed to weaker UV radiation fields and are less processed (Sloan et al. 2007). While we note that the amount of UV radiation background in our PPN sample is minimal, our results are consistent with the trends as reported by Sloan et al. (2007). Proposed explanations for the wavelength change of the AIB features include nitrogen-inclusion, variations in the fraction of carbon isotope, size distribution of the grains, ionization state, and the content ratio of aliphatic to aromatic bands (see, e.g., Bernard-Salas et al. 2009, and references therein). We suggest that this shift of the peaks of the AIB features to shorter wavelengths is related to the relative strengths of the aliphatic to aromatic components as well as growth of the sizes of the aromatic rings as the star evolve from PPN to PN (Kwok, Volk, \& Bernath 2001). Photochemistry may be the underlying cause of these changes.

The $11.3 \mu \mathrm{m}$ AIB feature is identified with the $\mathrm{C}-\mathrm{H}$ out-of-plane bending mode of aromatic compounds. However, when the number of rings in the aromatic units is small, there can be 2 (duo), 3 (trio), or 4 (quarto) adjacent $\mathrm{CH}$ groups and their out-of-plane bending mode frequencies can be slightly different (Hudgins \& Allamandola 1999). Generally, the duo- $\mathrm{CH}$ bending mode is in the $11.6-12.5 \mu \mathrm{m}$ range, the trio- $\mathrm{CH}$ mode is in the 
12.4-13.3 $\mu \mathrm{m}$, and the quarto- $\mathrm{CH}$ mode in the $13-13.6 \mu \mathrm{m}$ range. While the $11.3 \mu \mathrm{m}$ solo$\mathrm{CH}$ mode is dominant in PNs, the other modes are seen to be relatively stronger in PPNs (Kwok, Volk, \& Hrivnak 1999). This observation has led to the suggestion that the aromatic units grow in size as the star evolve from PPNs to PNs (Kwok 2004). With our present fitting exercise, it is possible to quantify this change. In Figure 5 we plotted the ratios of the sum of the strengths of the duo, tri, and quatro modes to the solo mode as a function of central star temperature. It can be seen that the strengths of the duo, trio and quatro modes decrease relative to the solo mode as the star evolves to higher temperatures, supporting the scenario that the aromatic units get larger with evolution.

The derived fluxes from each of the AIB features as well as their total contribution to the observed infrared fluxes are given in Table 2, Comparing the last column of Table 2 to the values in columns 8, 10, 12 in Table 1, we can see that the contribution of the AIB features to the total emitted infrared flux is much smaller than that of the $30 \mu \mathrm{m}$ feature and is of the same order (a few \%) as that of the $21 \mu \mathrm{m}$ feature. In some cases, the flux of the $11.3 \mu \mathrm{m}$ feature alone is larger than (e.g., in IRAS $05341+0852$ and IRAS 07430+1115), or about equal to (as in IRAS $22574+5435$ ) that of the $21 \mu \mathrm{m}$ feature .

Cerrigone et al. (2009) found that about $40 \%$ of their post-AGB star sample exhibit both AIBs and silicate features, and suggested that the spatial segregation of different dust populations in the envelopes can lead to the mixed chemistry and a disk/torus can serve as a reservoir preserving O-bearing molecules. However, we do not detect silicates features in our (C-rich) sources, suggesting that the $21 \mu \mathrm{m}$ sources do not favor the formation/survival of O-bearing compounds.

\section{3. $\quad 15-20 \mu \mathrm{m}$ plateau feature}

A $15-20 \mu \mathrm{m}$ plateau emission feature is commonly seen in PNs and other objects with a hot central star and has been attributed to $\mathrm{C}-\mathrm{C}-\mathrm{C}$ bending modes of aromatic rings (Van Kerckhoven et al. 2000). For example, this feature is seen in IRAS 06556+1623 and

IRAS 18442-1144 and several other post-AGB stars (Cerrigone et al. 2009). In most of our sources, this region is difficult to examine because of overlap with the blue wing of the 21 $\mu \mathrm{m}$ feature. However, in IRAS $01005+7910$ this is seen as a prominent feature, as shown in Figure 6. Integrating the fluxes between 15.2 and $20 \mu \mathrm{m}$, we obtain an integrated strength of $8.5 \times 10^{-13} \mathrm{~W} / \mathrm{m}^{2}$, leading to strength ratios relative to the $\mathrm{C}$-C stretching mode at $6.2 \mu \mathrm{m}$ of $22\left(I_{\text {plateau }} / I_{6.2}\right)$ and the $\mathrm{C}-\mathrm{H}$ out-of-plane bending model at $11.3 \mu \mathrm{m}$ of $31\left(I_{\text {plateau }} / I_{11.3}\right)$. The plateau/6.2,11.3 strength ratios are much larger than the values $(\leq 5)$ found in the sample of H II regions, Ae/Be stars, and PNs studied by Van Kerckhoven et al. (2000). Whether 
this is an evolutionary trend or particular to IRAS $01005+7910$ is difficult to say based on only one case. In this respect, it would be good if one could obtain $15-20 \mu \mathrm{m}$ spectra of other C-rich PPNs without a strong $21 \mu \mathrm{m}$ feature.

\section{4. $\quad 15.8 \mu \mathrm{m}$ feature}

In a previous paper (Hrivnak et al. 2009), we called attention to a moderately broad emission feature at $15.8 \mu \mathrm{m}$ seen in the Spitzer spectra of several carbon-rich PPNs with the $21 \mu \mathrm{m}$ feature. This feature is seen in the several additional spectra included in this study. While a weak feature at $15.9 \mu \mathrm{m}$ has been detected in the reflection nebula NGC 7023 (Werner et al. 2004) and some star-forming galaxies (see Fig. 5 of Smith et al. 2007), the feature seen in the PPNs in the present study is significantly broader. We noted there was a suggestion of a correlation with the strength of the $21 \mu \mathrm{m}$ feature in the sense that the two sources with the strongest $21 \mu \mathrm{m}$ features also had the strongest $15.8 \mu \mathrm{m}$ features (IRAS 06530-0230 and 23304+6147). We can examine this more quantitatively in this study with the measurement of the strength of this feature in these sources (see Table 2) and the inclusion of two other $21 \mu \mathrm{m}$ sources (IRAS 04296+4329 and IRAS 22223+4327).

Fig. 7 displays the $15.8 \mu \mathrm{m}$ fluxes versus the $21 \mu \mathrm{m}$ fluxes for the PPN sample. With the exception of IRAS $04296+3429$, there seems to be a positive correlation between the two fluxes, although more data, specially those of strong $21 \mu \mathrm{m}$ sources, are required to confirm this conjecture. Both 15.8 and $21 \mu \mathrm{m}$ features have an asymmetrical profile. In Fig. 8, we compare the profiles of the two features. While in all the cases the $15.8 \mu \mathrm{m}$ feature is narrower than the $21 \mu \mathrm{m}$ feature, they are similar in shape: both have a steeper blue rise and a shallower red tail.

\subsection{Molecular lines}

In addition to the above broad features, there are also molecular lines in the spectra. Hrivnak et al. (2009) have detected the $13.7 \mu \mathrm{m} \nu_{5}$ line of $\mathrm{C}_{2} \mathrm{H}_{2}$ in five PPNs, one (IRAS 22574+6609) in absorption, three (IRAS 05341+0852, IRAS 06530-0213, and IRAS $07430+1115$ ) in emission, and one (IRAS 23304+6147) in both absorption and emission (P Cygni profile). From our examination of the spectra, the $\mathrm{C}_{2} \mathrm{H}_{2}$ could also be present in emission in IRAS $04296+3429 . \mathrm{C}_{2} \mathrm{H}_{2}$ is the most abundant molecular species after $\mathrm{H}_{2}$ and $\mathrm{CO}$ in carbon-rich envelopes. In our sample, about $70 \% \mathrm{PPNs}$ exhibit the $\mathrm{C}_{2} \mathrm{H}_{2}$ feature. IRAS $05341+0652$ has the strongest $\mathrm{C}_{2} \mathrm{H}_{2}$ emission. We do not find correlation between the 
fluxes of $\mathrm{C}_{2} \mathrm{H}_{2}$ and those of other features.

Furthermore, we can unambiguously identify the $\mathrm{H}_{2} 0-0 \mathrm{~S}(0)$ line at $28 \mu \mathrm{m}$ in IRAS $04296+3429$ and IRAS 22223+4327 and their spectra are shown in Figure. 9. However, the expected next strongest $\mathrm{H}_{2}$ line ortho ground-state $v=0-0 \mathrm{~S}(1)$ line at $17 \mu \mathrm{m}$ is not detected. We do not find any correlation between the strengths of dust features and those of molecular hydrogen emission. IRAS 04296+3429 shows relatively strong AIB and $21 \mu \mathrm{m}$ features, but these features are weak in the spectrum of IRAS $22223+4327$. Table 4 gives the fluxes of the $\mathrm{S}(0)$ transition and the flux upper limits of the $\mathrm{S}(1)$ transition estimated from the $3 \sigma$ flux noise level. These values allow us to determine the upper limits of excitation temperatures in the two objects. Under an assumption of local thermodynamic equilibrium (LTE), we have

$$
\frac{N_{v, J}}{g_{J}}=N_{\text {tot }} \frac{\exp \left(-E_{v, J} / k T_{\mathrm{ex}}\right)}{\Sigma_{v^{\prime}, J^{\prime}} g_{J^{\prime}} \exp \left(-E_{v^{\prime}, J^{\prime}} / k T_{\mathrm{ex}}\right)},
$$

where $N_{v, J}$ is the population of the upper level $(v, J), g_{J}$ is the level degeneracy under the assumption of ortho/para ratio $=3,\left(g_{J}=(2 J+1)\right.$ for even $J$ and $3(2 J+1)$ for odd $\left.J\right), N_{\text {tot }}$ is the total $\mathrm{H}_{2}$ density, $E_{v, J}$ is the energy of the upper level, $T_{\mathrm{ex}}$ is the excitation temperature, and $k$ is Boltzmann's constant. The flux of a given transition, $F$, is proportional to $N_{v, J}$ ( $F \propto N_{v, J} A / \lambda$, where $A$ is the radiative transition probability). From Equation (1) we have

$$
T_{\mathrm{ex}}=\frac{E_{v, J_{1}} / k-E_{v, J_{0}} / k}{\ln \left(F_{0} \lambda_{0} g_{J_{1}} A_{1}\right)-\ln \left(F_{1} \lambda_{1} g_{J_{0}} A_{0}\right)},
$$

where the subscripts, 0 and 1 , represent the transitions $\mathrm{S}(0)$ and $\mathrm{S}(1)$, respectively. Taking the $E_{v, J}$ and $A$ values from Rosenthal et al. (2000), we derive the $T_{\text {ex }}$ upper limits of $56 \mathrm{~K}$ and $51 \mathrm{~K}$ for IRAS $04296+3429$ and IRAS 22223+4327, respectively. The extremely low excitation temperatures of $\mathrm{H}_{2}$ seem to suggest that the contributions from UV pumping and shock waves to the excitation of $\mathrm{H}_{2}$ are insignificant in the two PPNs.

This is consistent with previous observations of the $\mathrm{H}_{2} v=1-0 \mathrm{~S}(1)$ line at $2.12 \mu \mathrm{m}$ of these two and other PPNs (Kelly \& Hrivnak 2005). It was found that $\mathrm{H}_{2}$ emission was seen in F-G spectral type PPNs only if they were clearly bipolar in morphology with a relatively dense equatorial dust torus and a bipolar outflow, and that the $\mathrm{H}_{2}$ emission in these cases was collisionally excited.

\section{Concluding remarks}

We have investigated the mid-IR spectral features - AIBs, $15-20 \mu \mathrm{m}$ plateau, $15.8 \mu \mathrm{m}$, $21 \mu \mathrm{m}$, and $30 \mu \mathrm{m}$ - in a sample of PPNs using their Spitzer/IRS spectra. The strengths of 
these emission features are determined using a decomposition method. We find that the 21 and $30 \mu \mathrm{m}$ features are strong in these PPNs, contributing up to 5 and $30 \%$, respectively, of the total infrared fluxes observed. A prominent $15-20 \mu \mathrm{m}$ broad emission plateaus is detected in IRAS $01005+7910$. The $15.8 \mu \mathrm{m}$ emission feature, seen in almost all our objects, is found to have similar intrinsic emission profile as the $21 \mu \mathrm{m}$ feature. Molecular hydrogen lines are observed in two of the PPNs, showing the presence of the neutral circumstellar component. Most of the PPNs require two dust components to fit their underlying continua. We suggest that the cold component is due to an extended dust halo probably left over from the AGB phase, and a warmer component is due to a dust torus in the central region of the PPN. This suggestion can be tested by future infrared imaging observations.

The fact that the duo, trio, and quarto $\mathrm{C}-\mathrm{H}$ out-of-plane bending modes decline in strength with evolution relative to the solo mode is consistent with the suggestion that the aromatic component grows in size as the star evolves to higher temperatures. There is also evidence for the gradual shift of the peak of the $11.3 \mu \mathrm{m}$ feature to the blue with evolution. The 21 and $30 \mu \mathrm{m}$ features, which carriers are unknown, are shown to decline in strength with evolution. These observations will help us to constrain future chemical evolution of organic synthesis in the late stages of stellar evolution.

This work was supported by a grant to SK from the Research Grants Council of the Hong Kong Special Administrative Region, China (Project No. HKU 7020/08P) and a grant to YZ from the Seed Funding Programme for Basic Research in HKU (200909159007).

\section{A. Principal component analysis}

We also investigated the variety of the PPN spectra using the method of principal component analysis (PCA). PCA is a mathematical tool which has been commonly applied to spectral classification (e.g. Francis et al. 1992; Suzuki 2006, and references therein). The basic idea of PCA is to obtain new independent variables, called the principal components (PC), through orthogonal transformation. The new variables are a linear combination of the original data. The relative contributions to the spectrum variance of each $\mathrm{PC}$ can be determined. The PCA technique can reduce the multidimensionality of the data and enables one to study the correlations between different spectral properties (see, Francis et al. 1992, for a more detailed description of PCA). This analysis has the potential to give new insight into the spectral features.

In our work, the common wavelength coverage $(10-35 \mu \mathrm{m})$ of the ten PPN spectra was

considered for PCA. The formulation of PCA can be found in many papers (e.g. Ronen et al. 
1999). We first subtracted the continuum and normalized each spectrum by its integrated flux. The mean spectrum is obtained by

$$
<F\left(\lambda_{j}\right)>=\frac{1}{N} \sum_{i=1}^{N} F_{i}\left(\lambda_{j}\right),
$$

where $N=10$ is the number of the PPN spectra, and $j=1, \ldots, M$ is the number of the wavelength channels. The data set can be denoted by a $N \times M$ matrix

$$
X_{i j}=F_{i}\left(\lambda_{j}\right)-<F\left(\lambda_{j}\right)>.
$$

The eigenvector and eigenvalue of its covariance matrix, respectively, give the new axis (PCs) and the variance along the new axis. The PCs are ordered according to their relative contributions to the spectrum variance, such that the first PC accounts for the maximum variance.

Figure 10 shows the mean spectrum and the first three PCs (PC1, PC2, and PC3). The first three PCs account for $79 \%$ of the variance in the spectra. The mean spectrum mainly exhibits three features: the AIBs around 10-15 $\mu \mathrm{m}$, the $21 \mu \mathrm{m}$ feature, and the $30 \mu \mathrm{m}$ feature. PC1 accounts for $37 \%$ of the variance. Compared to other PCs, the most prominent feature of PC1 is the dip around $18 \mu \mathrm{m}$. Thus, it can modulates the strengths of the $21 \mu \mathrm{m}$ feature and the emission from $12-15 \mu \mathrm{m}$. PC2 accounts for $29 \%$ of the variance. It displays the modulation of the AIB features from $10-15 \mu \mathrm{m}$, and especially the $11.3 \mu \mathrm{m}$ feature. Since PC2 can also modulate the flux of $12-15 \mu \mathrm{m}$ emission, PC1 particularly displays the modulation in the flux of the $21 \mu \mathrm{m}$ feature. PC3 account for 13 percent of the variance. It reflects the variation in the relative fluxes of individual AIB features. We can conclude that the variance in this spectrum sample is mainly caused by the $21 \mu \mathrm{m}$ and AIB features, but not by the $30 \mu \mathrm{m}$ feature.

Figure 11] shows the projections of the PPN spectra on to the first two PCs (denoted by $\mathrm{pc} 1$ and pc2). The values of $\mathrm{pc} 1$ and $\mathrm{pc} 2$ represent the contribution of each component to the difference between individual PPN spectrum and the mean spectrum. We can find that the non- $21 \mu \mathrm{m}$ source, IRAS 01005+7910, has the lowest pc1 value, and the strongest $21 \mu \mathrm{m}$ source (IRAS 06530-0213, IRAS 23304+6147, IRAS 04296+3429) have the largest pc1 values. If the $21 \mu \mathrm{m}$ feature arises from the warm torus(see Section 4), the value of pc1 could represent the content of warm dust in the torus. The value of pc2 reflects the strengths of AIBs with respect to the mean spectrum. The sources with weak AIB features (IRAS 22223+4327 and IRAS 19477+2401) have the largest pc2 values while the sources with strong AIB features (IRAS 05341-0852, IRAS 22574+6609) have the lowest pc2 values. Therefore, PCA 
allows us to objectively and quantitatively classify the spectra of PPNs. However, the current sample is too small for the analysis of higher-order PCs. With a larger PPN sample, it would be intriguing to use PCA to study the global spectral behavior and the relation between the fluxes of different AIB features.

\section{REFERENCES}

Bernard-Salas, J., Pottasch, S. R., Gutenkunst, S., Morris, P. W., \& Houck, J. R. 2008, ApJ, 672,274

Bernard-Salas, J. et al. 2009, ApJ, 699, 1541

Cerrigone, L., Hora, J.L., Umana, G., Trigilio, C. 2009, ApJ, 703, 585

Forrest, W. J., Houck, J. R., \& McCarthy, J. F. 1981, ApJ, 248, 195

Francis, P. J., Hewett, P. C., Foltz, C. B., \& Chaffee, F. H. 1992, ApJ, 398, 476

Geballe, T. R., Tielens, A. G. G. M., Kwok, S., \& Hrivnak, B. J. 1992, ApJ, 387, L89

Goebel, J. H., \& Moseley, S. H. 1985, ApJ, 290, L35

Henry, R. B. C., Skinner, J. N., Kwitter, K. B., \& Milingo, J. B. 2006, in IAU Symp. 234 Planetary Nebulae, eds. M. J. Barlow, \& R. H. Méndez (Cambridge: Cambridge University Press), P.417

Hony, S., Waters, L. B. F. M., \& Tielens, A. G. G. M. 2002, A\&A, 390, 553

Houck, J. R., et al. 2004, ApJS, 154, 18

Hrivnak, B. J., Volk, K., Geballe, T. R., \& Kwok, S. 2008, in IAU Symp. 251: Organic Matter in Space, ed. S. Kwok, S. Sandford (Cambridge: Cambridge University Press), 213

Hrivnak, B. J., Volk, K., \& Kwok, S. 2009, ApJ, 694, 1147

Hu, A., \& Duley, W.W. 2008, ApJ, 677, L153

Hudgins, D.M., Allamandola, L.J. 1999, ApJ, 516, L41

Jones, A. P., Duley,W.W. \& Williams, D. A. 1990, Q. J. R. Astron. Soc. 31, 567

Kelly, D.M., \& Hrivnak, B. J. 2005, ApJ, 629, 1040

Keller, L.D. et al. 2008 ApJ, 684, 411 
Kwok, S. 1993, ARAA, 31, 63

Kwok, S. 2004, Nature, 430, 985

Kwok, S. 2009, Ap\&SS, 319, 5

Kwok, S., Volk, K., \& Bernath, P. 2001, ApJ, 554, L87

Kwok, S., Volk, K., \& Bidelman, W.P. 1997, ApJS, 112, 557

Kwok, S., Volk, K., \& Hrivnak, B. J. 1989, ApJ, 345, L51

Kwok, S., Volk, K.M., \& Hrivnak, B.J. 1999, A\&A, 350, L35

Kwok, S., Volk, K., \& Hrivnak, B.J. 2002, ApJ, 573, 720

Meixner, M, Zalucha, A., Ueta, T., Fong, D., \& Justtanont, K. 2004, ApJ, 614, 371

Nakashima, J., Koning, N., Kwok, S., \& Zhang, Y. 2009, ApJ, 692, 402

Papoular, R. 2001, A\&A, 378, 597

Peeters, E., et al. 2002, A\&A, 390, 1089

Pendleton, Y.J., \& Allamandola, L.J. 2002, ApJS, 138, 75

Pottasch, S. R., \& Bernard-Salas, J. 2006, ApJ, 457, 189

Rosenthal, D., Bertoldi, F., \& Drapatz, S. 2000, A\&A, 356, 705

Posch, Th., Mutschke, H., \& Andersen, A. 2004, ApJ, 616, 1167

Ronen, S., Aragón-Salamanca, A., \& Lahav, O. 1999, MNRAS, 303, 284

Sakata, A., Wada, S., Okutsu, Y., Shintani, H., \& Nakada, Y. 1983, Nature, 301, 493

Scott, A.D., Duley, W.W., \& Jahani, H.R. 1997, ApJ, 490, L177

Sloan, G. C., Kraemer, K. E., Price, S. D., \& Shipman, R. F. 2003, ApJS, 147, 379

Sloan, G. C. et al. 2007, ApJ, 664, 1144

Smith, J. D. T. et al. 2007, ApJ, 656, 770

Speck, A.K., \& Hofmeister, A.M. 2004, ApJ, 600, 986

Suzuki, N., 2006, ApJ, 163, 110 
Van Kerckhoven, C. et al. 2000, A\&AS, 357, 1013

Van Winckel, H. 2003 ARAA, 41, 391

Volk, K., Kwok, S., \& Hrivnak, B. J. 1999, ApJ, 516, L99

Volk, K., Kwok, S., Hrivnak, B. J., \& Szczerba, R. 2002, ApJ, 567, 412

Volk, K., Matsuura, M, Bernard-Salas, J., et al. 2010, Bull. AAS, 42, 486

Werner, M., et al. 2004, ApJS, 154, 1

Zhang, K, Jiang, B. W., \& Li, A. 2009, ApJ, 702, 680

This preprint was prepared with the AAS LATEX macros v5.2. 

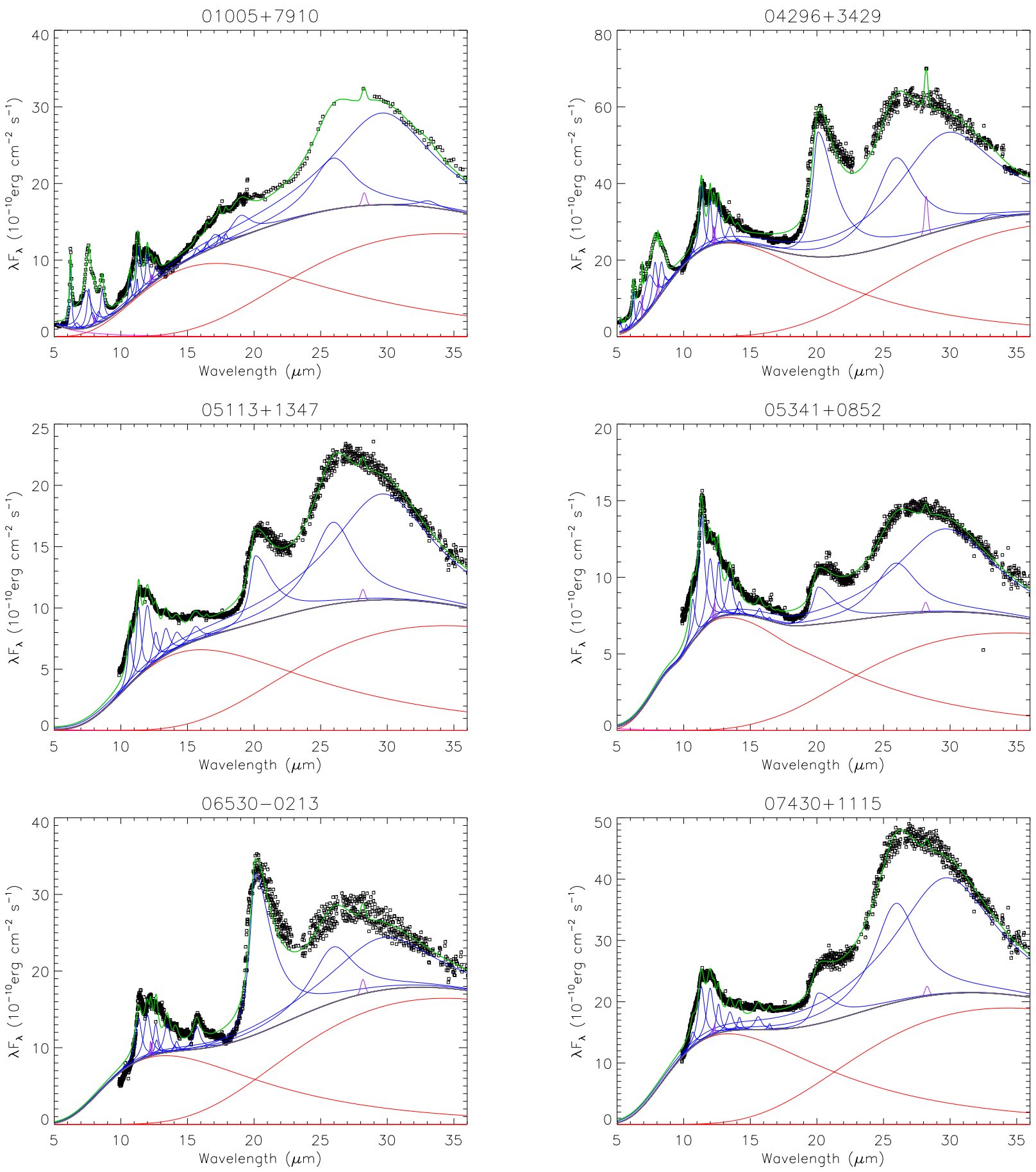

Fig. 1.- Decomposition of the IRS spectra. The black squares are the observations. The red solid lines show the thermal dust components. The stellar continuum, although included in the plots, are too weak to be seen. The gray lines show the total of the stellar and dust continuum components. The dust features and $\mathrm{H}_{2}$ lines are indicated by the blue and violet lines, respectively. The green solid lines are total model spectra. 

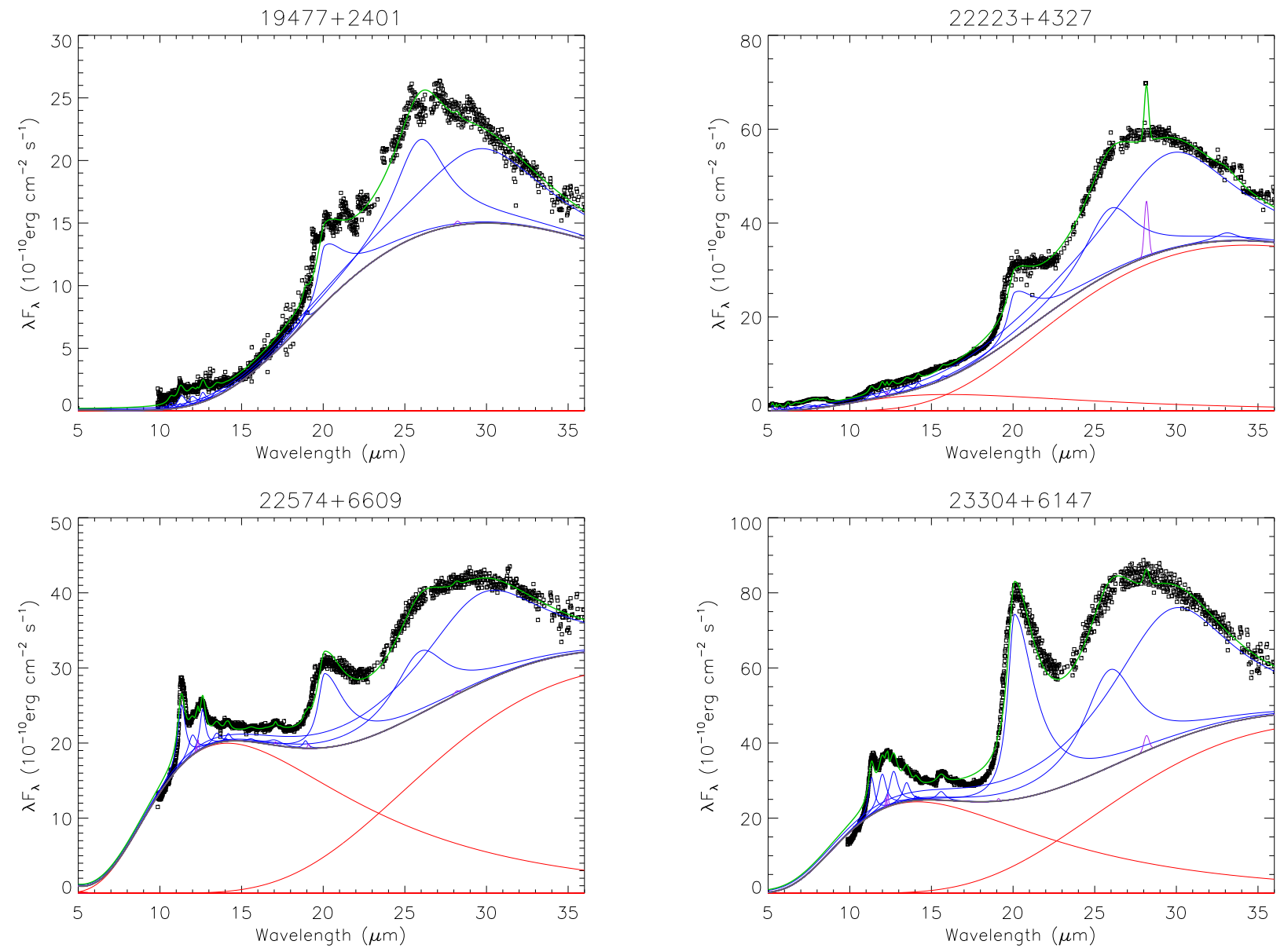

Fig. 1.- continued. 


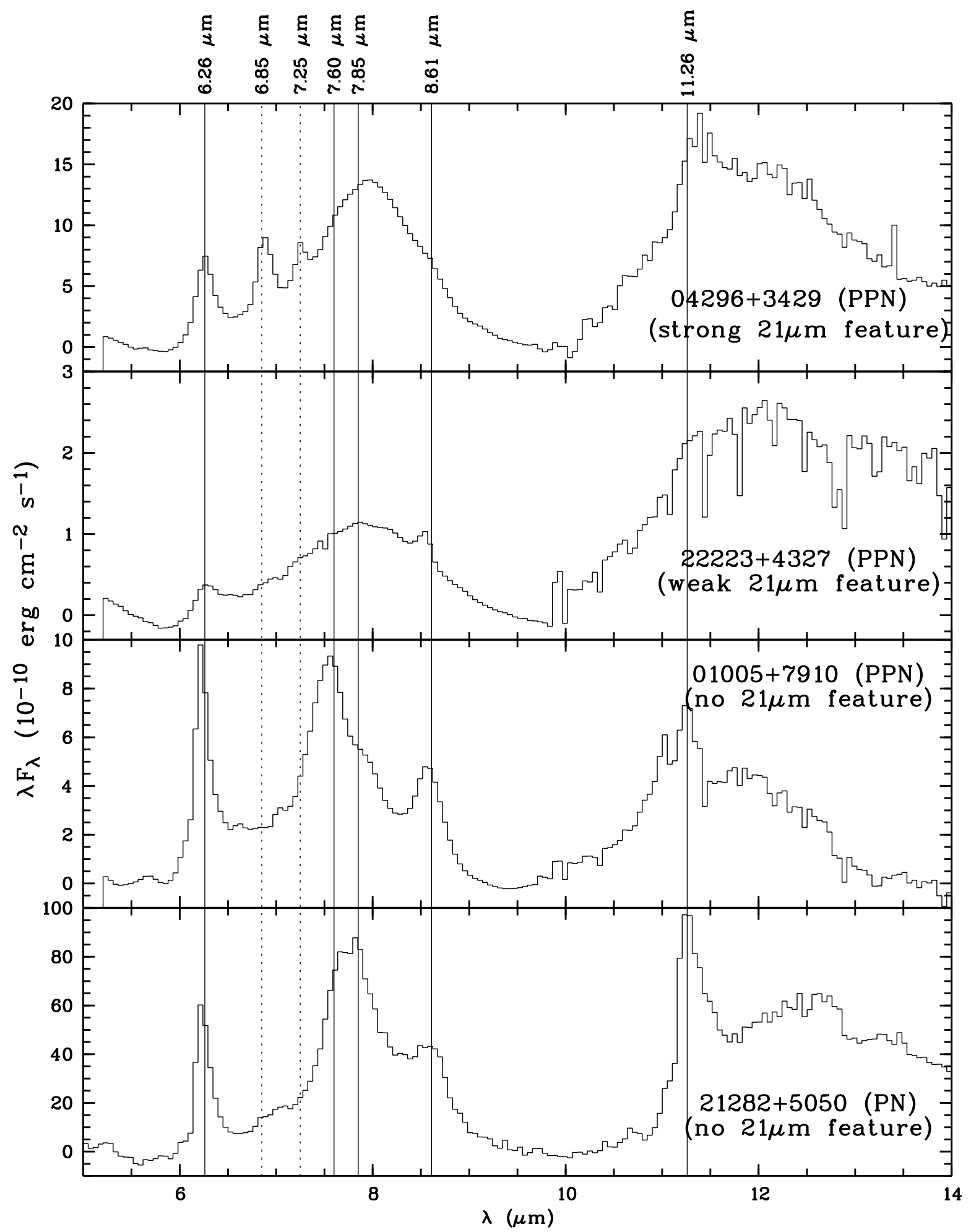

Fig. 2.- Continuum-subtracted IRS spectra. The continuum is the sum of the model stellar and dust continuum components. The solid and dash lines indicate the positions of aromatic and aliphatic emission features, respectively. 


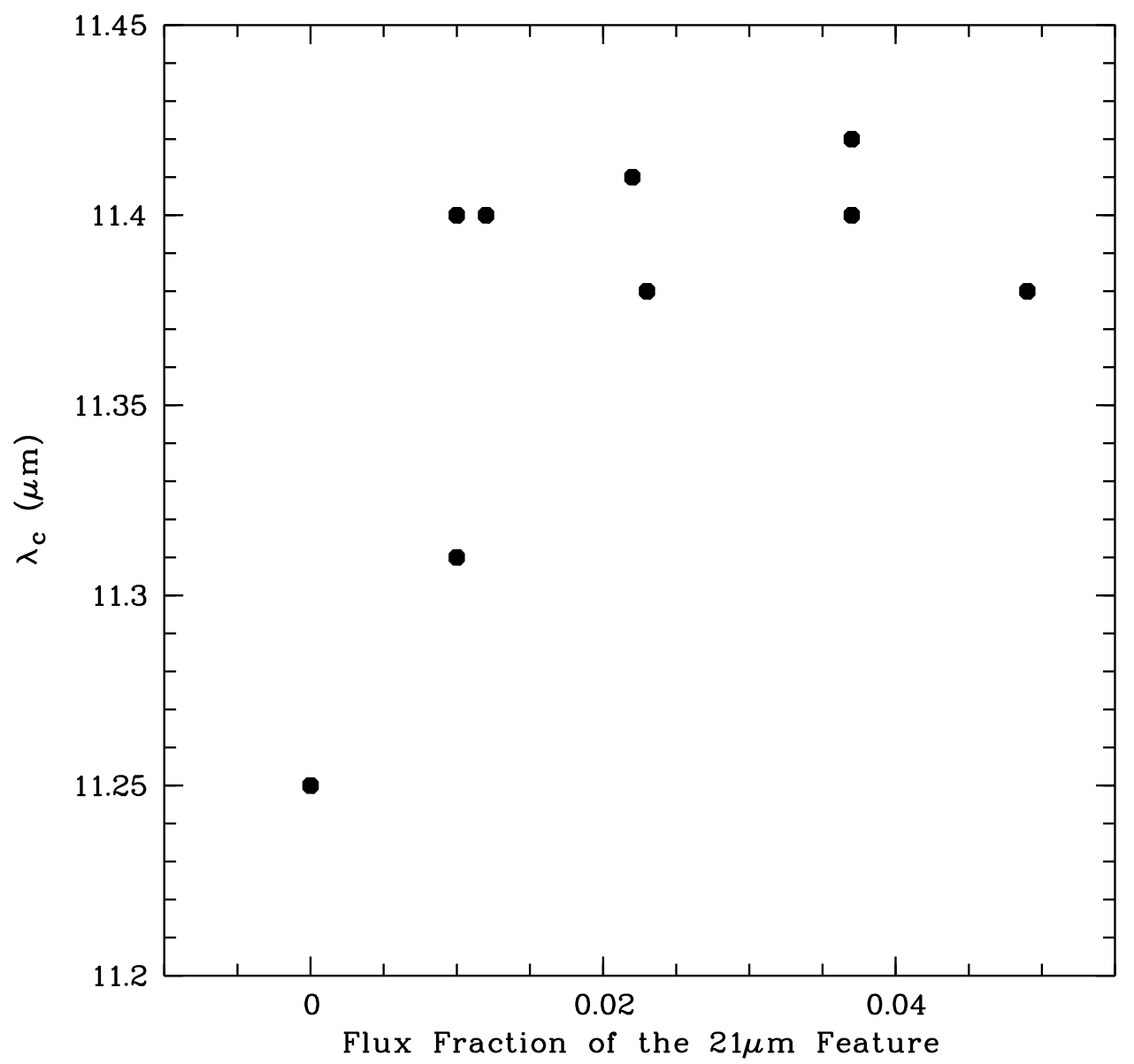

Fig. 3.- Peak wavelengths of the $11.3 \mu \mathrm{m}$ feature versus flux fractions of the $21 \mu \mathrm{m}$ feature. The object with zero $21 \mu \mathrm{m}$ flux is IRAS $01005+7910$. 


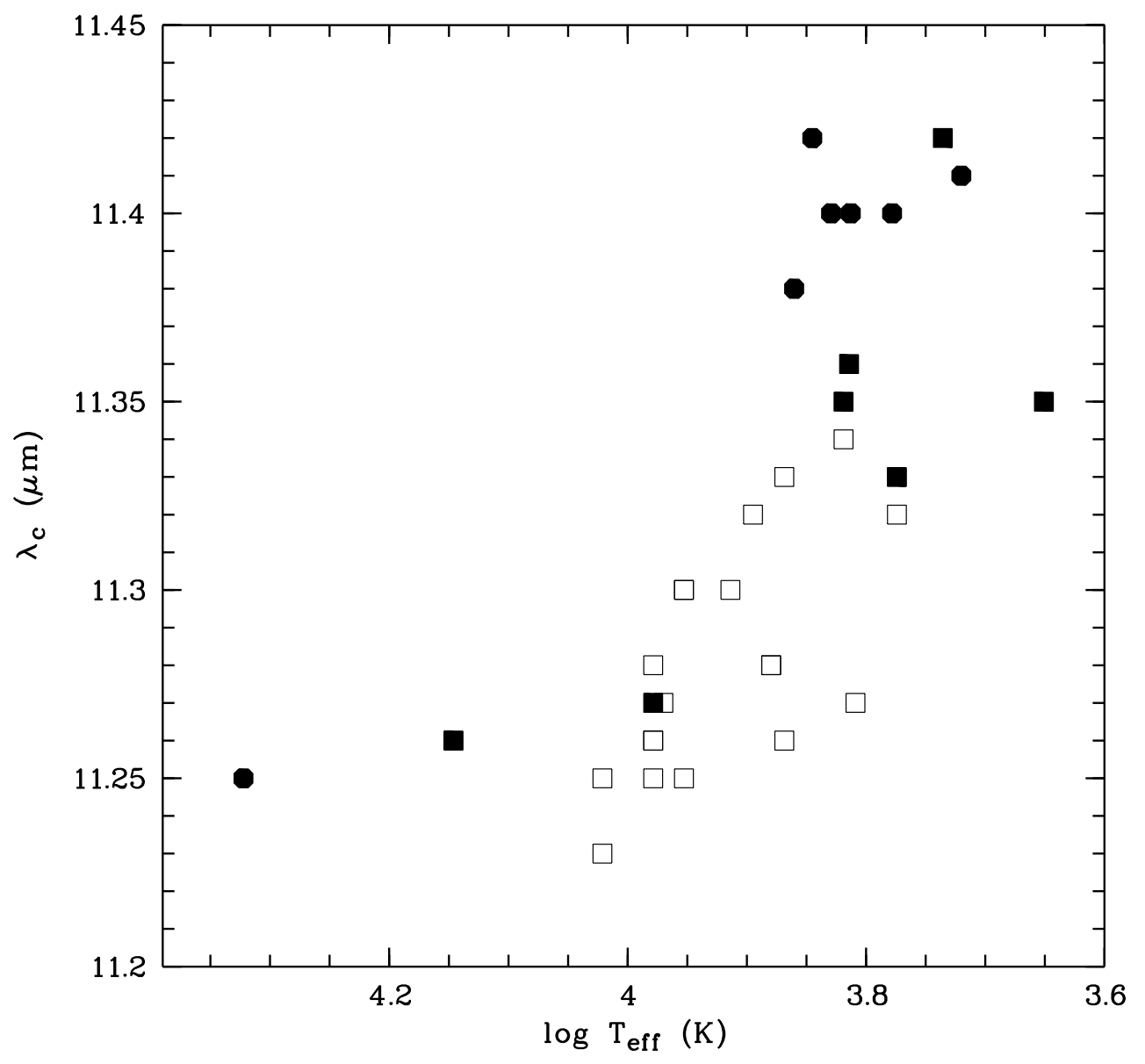

Fig. 4.- Peak wavelength of the $11.3 \mu \mathrm{m}$ feature versus effective temperatures of the central stars. The filled squares are objects from Sloan et al. (2007), the open squares are Ae/Be stars from Keller et al. (2008) and the filled circles are PPNs in the present study. 


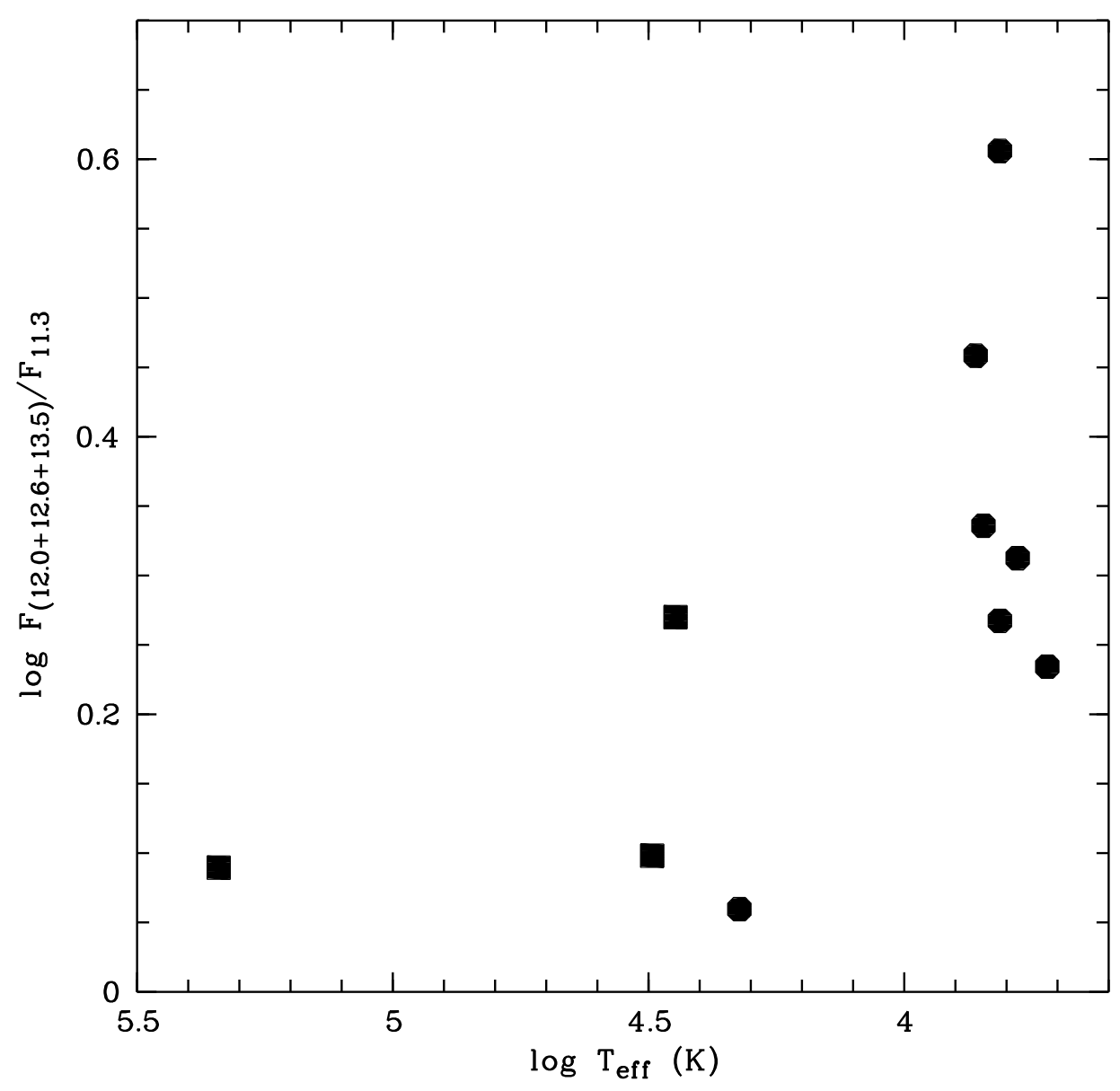

Fig. 5.- Ratio of the sum of the strengths of the 12.0, 12.6, and $13.5 \mu \mathrm{m}$ features to the strength of the $11.3 \mu \mathrm{m}$ feature as a function of central star temperature. The filled circles are PPNs in our sample, and the filled squares are (from right to left) PNs IRAS 21282+5050, BD+303639, and NGC 7027. 


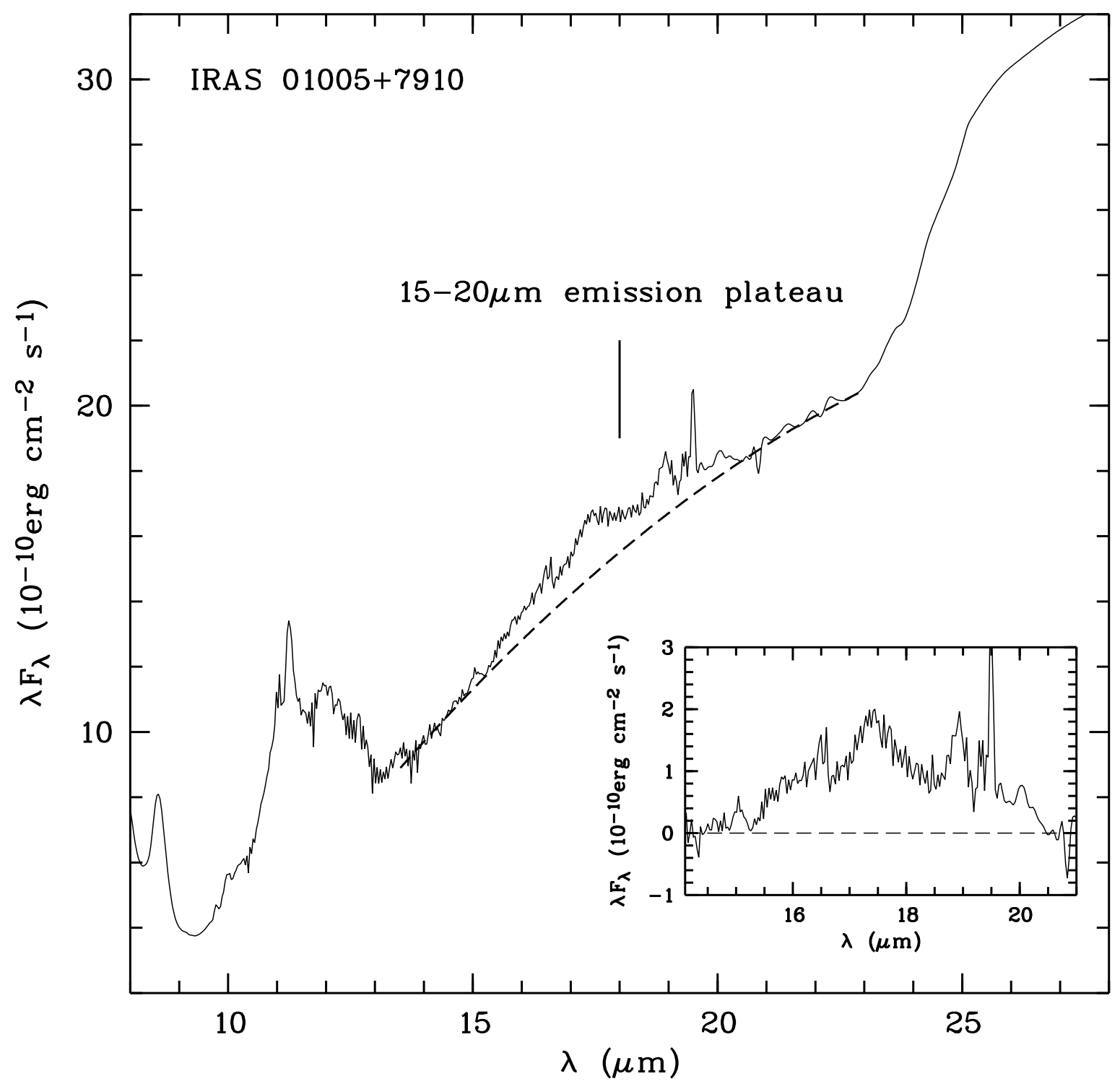

Fig. 6.- The 15-20 $\mu \mathrm{m}$ emission plateau. The dashed line indicates the continuum. The continuum-subtracted spectrum is shown in the lower right panel. 


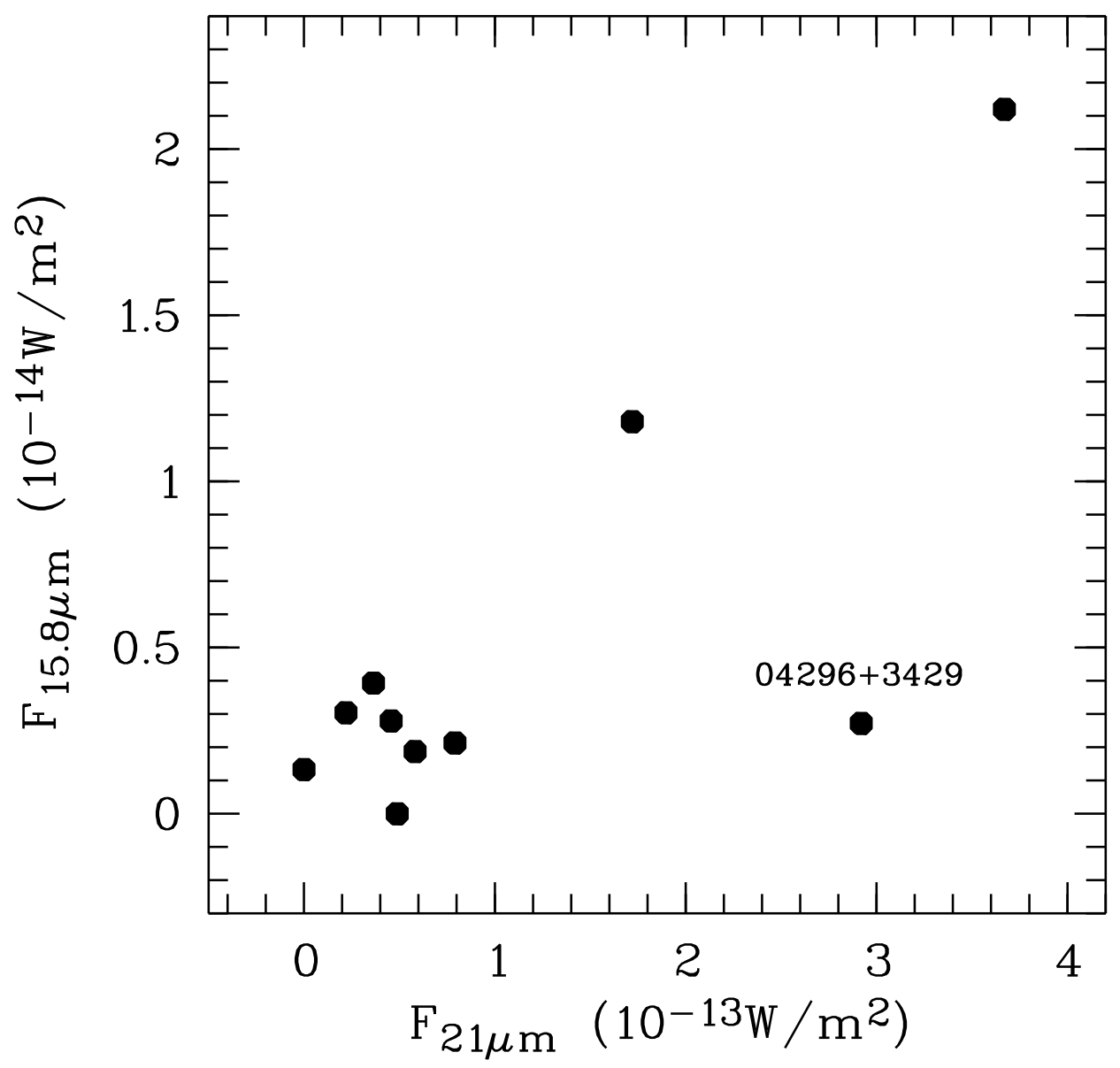

Fig. 7.- The strength of the $15.8 \mu \mathrm{m}$ feature versus the strength of the $21 \mu \mathrm{m}$ feature. 


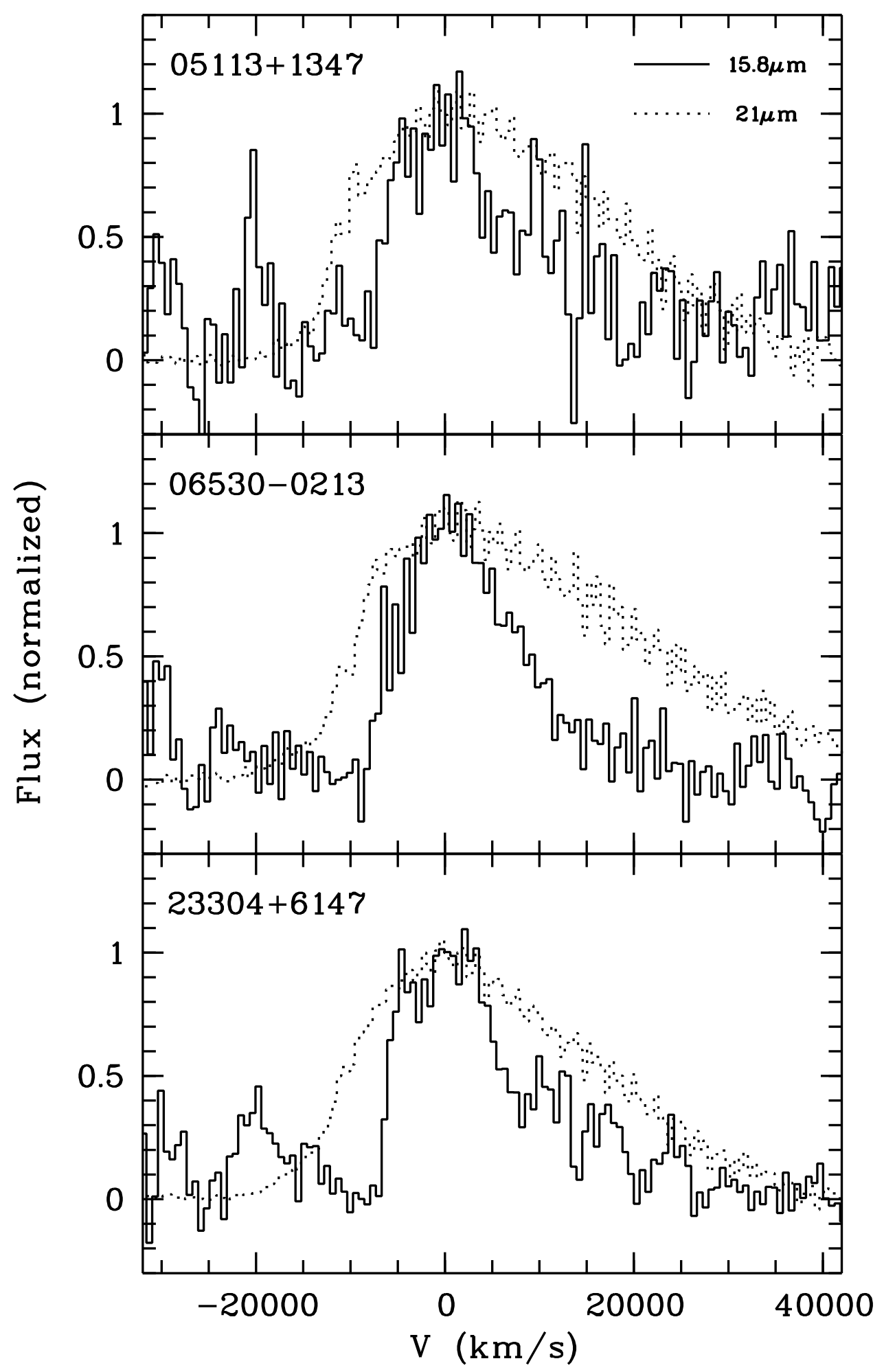

Fig. 8. - The profiles of the 15.8 and $21 \mu \mathrm{m}$ features in three PPNs. 


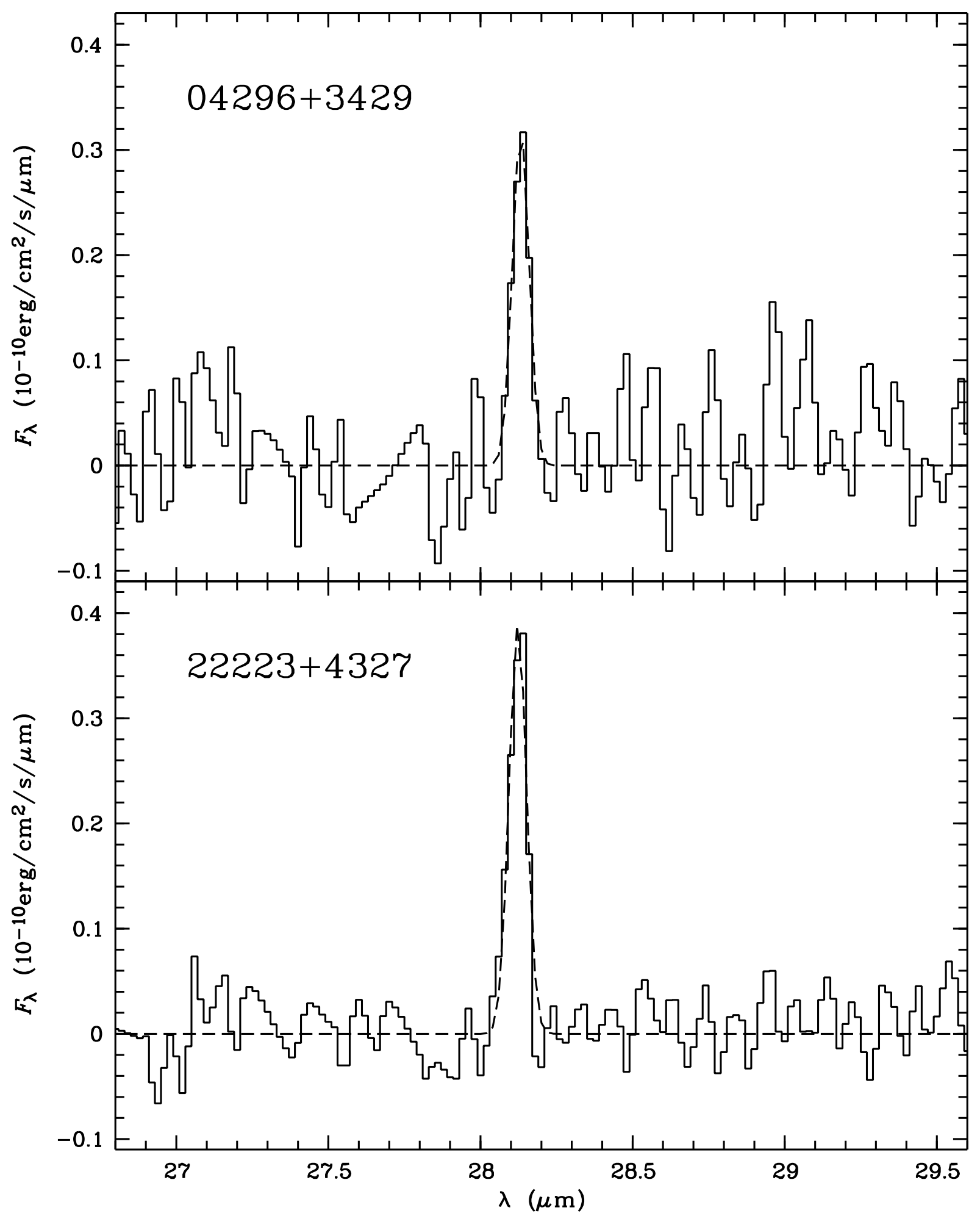

Fig. 9.- The observed profiles of the $\mathrm{H}_{2} 0-0 \mathrm{~S}(0)$ transitions in IRAS $04296+3429$ and IRAS 22223+4327. The dashed lines represent a Gaussian fit. 


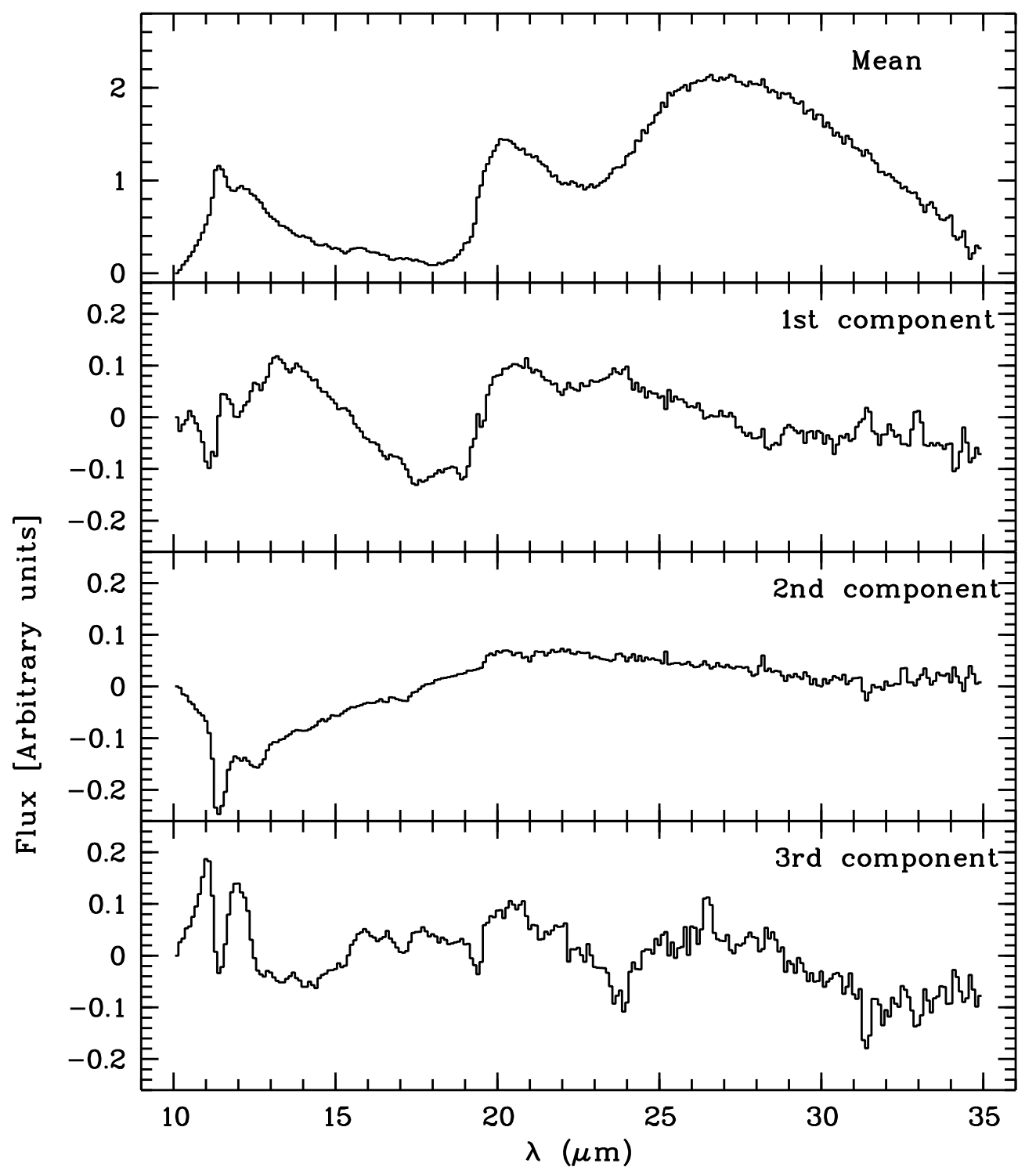

Fig. 10.- The mean spectrum and the first three PCs. 


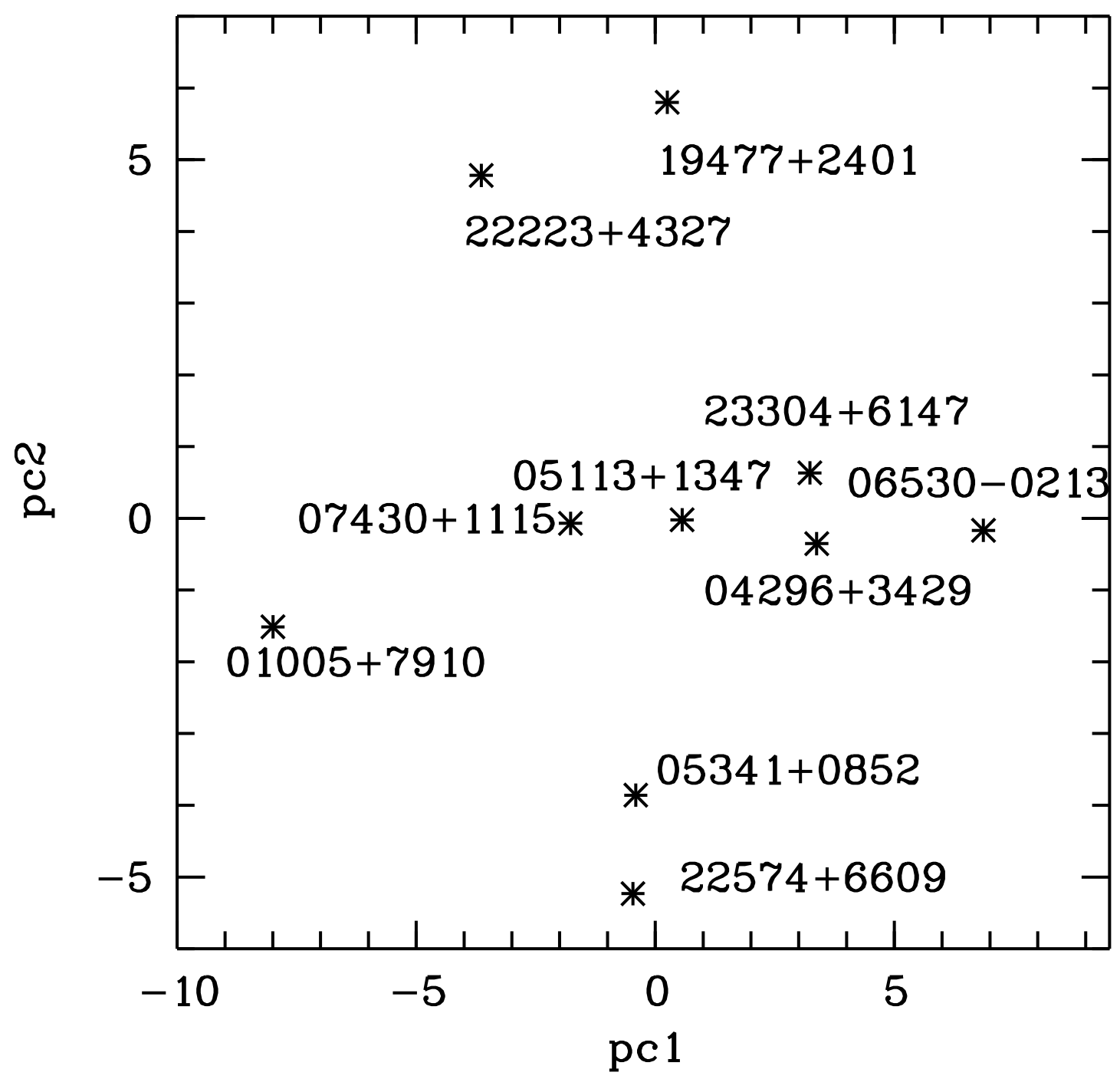

Fig. 11. - Projections on to the first and the second eigenvectors of the spectrum sample. 
Table 1. Effective temperatures and integrated intensities of the dust continuum and the 21 and $30 \mu \mathrm{m}$ features.

\begin{tabular}{|c|c|c|c|c|c|c|c|c|c|c|c|}
\hline \multirow{3}{*}{$\begin{array}{c}\text { Object } \\
\text { IRAS ID }\end{array}$} & \multirow{3}{*}{$\begin{array}{c}\text { Stellar } \\
\text { Temperature }^{a} \\
(\mathrm{~K})\end{array}$} & \multicolumn{4}{|c|}{ Dust Continuum } & \multicolumn{6}{|c|}{ Dust Features ${ }^{b}$} \\
\hline & & \multicolumn{2}{|c|}{ Warm } & \multicolumn{2}{|c|}{ Cold } & \multicolumn{2}{|c|}{$20.1 \mu \mathrm{m}$} & \multicolumn{2}{|c|}{$26 \mu \mathrm{m}$} & \multicolumn{2}{|c|}{$30 \mu \mathrm{m}$} \\
\hline & & $(\mathrm{K})$ & $\left(\mathrm{W} / \mathrm{m}^{2}\right)$ & $\overline{(\mathrm{K})}$ & $\left(\mathrm{W} / \mathrm{m}^{2}\right)$ & $\left(\mathrm{W} / \mathrm{m}^{2}\right)$ & $(\%)^{c}$ & $\left(\mathrm{~W} / \mathrm{m}^{2}\right)$ & $(\%)^{c}$ & $\left(\mathrm{~W} / \mathrm{m}^{2}\right)$ & $(\%)^{c}$ \\
\hline $01005+7910$ & 21000 & 140 & $1.12 \mathrm{E}-12$ & 70 & $1.38 \mathrm{E}-12$ & $0.00(0.00) \mathrm{E}-00$ & 0.0 & $1.30(0.15) \mathrm{E}-13$ & 4.0 & $5.47(0.21) \mathrm{E}-13$ & 16.7 \\
\hline $04296+3429$ & 7000 & 180 & $2.39 \mathrm{E}-12$ & 60 & $3.15 \mathrm{E}-12$ & $2.92(0.01) \mathrm{E}-13$ & 3.7 & $6.19(0.04) \mathrm{E}-13$ & 7.9 & $1.12(0.01) \mathrm{E}-12$ & 14.3 \\
\hline $05113+1347$ & 5250 & 150 & $6.49 \mathrm{E}-13$ & 70 & $8.90 \mathrm{E}-13$ & $4.57(0.06) \mathrm{E}-14$ & 2.2 & $1.57(0.04) \mathrm{E}-13$ & 7.5 & $4.12(0.11) \mathrm{E}-13$ & 19.7 \\
\hline $05341+0852$ & 6500 & 180 & $6.46 \mathrm{E}-13$ & 70 & $7.52 \mathrm{E}-13$ & $2.20(0.06) \mathrm{E}-14$ & 1.2 & $9.25(0.35) \mathrm{E}-14$ & 5.1 & $1.88(0.08) \mathrm{E}-13$ & 10.3 \\
\hline $07430+1115$ & 6000 & 180 & $3.13 \mathrm{E}-13$ & 70 & $2.22 \mathrm{E}-12$ & $3.64(0.06) \mathrm{E}-14$ & 1.0 & $4.09(0.04) \mathrm{E}-13$ & 10.8 & $6.53(0.08) \mathrm{E}-13$ & 17.2 \\
\hline $19477+2401$ & $\ldots^{d}$ & $\ldots$ & $\ldots$ & 80 & $1.62 \mathrm{E}-12$ & $4.89(0.06) \mathrm{E}-14$ & 2.3 & $1.82(0.04) \mathrm{E}-13$ & 8.7 & $2.19(0.08) \mathrm{E}-13$ & 10.5 \\
\hline $22223+4327$ & 6500 & 150 & $4.03 \mathrm{E}-13$ & 70 & $3.89 \mathrm{E}-12$ & $7.91(0.09) \mathrm{E}-14$ & 1.4 & $3.66(0.04) \mathrm{E}-13$ & 6.6 & $7.30(0.07) \mathrm{E}-13$ & 13.2 \\
\hline $22574+6609$ & $\ldots e$ & 170 & $2.07 \mathrm{E}-12$ & 60 & $3.25 \mathrm{E}-12$ & $5.82(0.01) \mathrm{E}-14$ & 1.0 & $1.53(0.04) \mathrm{E}-13$ & 2.5 & $3.30(0.08) \mathrm{E}-13$ & 5.5 \\
\hline $23304+6147$ & 6750 & 170 & $2.56 \mathrm{E}-12$ & 60 & $5.07 \mathrm{E}-12$ & $3.67(0.01) \mathrm{E}-13$ & 3.7 & $5.78(0.04) \mathrm{E}-13$ & 5.8 & $1.10(0.01) \mathrm{E}-12$ & 11.1 \\
\hline
\end{tabular}

${ }^{a}$ Based on published model atmosphere studies.

${ }^{\mathrm{b}}$ The numbers in brackets are fitting uncertainties.

${ }^{\mathrm{c}}$ The flux fractions of the features to the total IR emission, which includes the contribution from the dust features from 10-35 $\mu \mathrm{m}$ and the dust thermal continuum. ${ }^{\mathrm{d}} \mathrm{G} 0$ spectral type.

eA1 spectral type. 
Table 2. Strengths of AIB and $15.8 \mu \mathrm{m}$ features. ${ }^{a}$

\begin{tabular}{|c|c|c|c|c|c|c|c|c|c|c|c|c|c|}
\hline IRAS ID & $6.2 \mu \mathrm{m}$ & $6.9 \mu \mathrm{m}$ & $7.4 \mu \mathrm{m}$ & $7.8 \mu \mathrm{m}$ & $8.3 \mu \mathrm{m}$ & $8.6 \mu \mathrm{m}$ & $10.7 \mu \mathrm{m}$ & $11.3 \mu \mathrm{m}$ & $12.0 \mu \mathrm{m}$ & $12.6 \mu \mathrm{m}$ & $13.5 \mu \mathrm{m}$ & $15.8 \mu \mathrm{m}$ & $\%$ of total ${ }^{c}$ \\
\hline $1005+7910$ & $3.84(0.12)$ & $0.73(0.02)$ & $9.58(0.40)$ & $1.58(0.23)$ & $1.32(0.18)$ & $2.72 \mathrm{E}(0.14)$ & $1.03(0.20)$ & $2.75(0.38)$ & $2.92(0.33)$ & $0.23(0.06)$ & $0.00(0.00)$ & $0.13(0.01)$ & 8.2 \\
\hline $4296+3429$ & $3.71(0.12)$ & $4.82(0.19)$ & $17.70(3.00)$ & $8.70(0.19)$ & $6.68(0.18)$ & $2.29 \mathrm{E}(0.14)$ & $1.09(0.23)$ & $8.26(0.27)$ & $8.54(0.34)$ & $6.21(0.31)$ & $3.15(0.25)$ & $0.27(0.02)$ & 9.1 \\
\hline $5113+1347$ & $\ldots^{b}$ & $\ldots$ & $\ldots$ & $\ldots$ & $\ldots$ & $\ldots$ & $0.18(0.02)$ & $2.25(0.28)$ & $3.28(0.33)$ & $0.58(0.02)$ & $0.00(0.00)$ & $0.28(0.02)$ & $>3.1$ \\
\hline $5341+0852$ & $\ldots$ & $\ldots$ & $\ldots$ & $\ldots$ & $\ldots$ & $\ldots$ & $0.23(0.02)$ & $3.62(0.28)$ & $3.00(0.34)$ & $2.23(0.33)$ & $1.47(0.27)$ & $0.30(0.02)$ & $>5.9$ \\
\hline $6530-0213$ & $\ldots$ & $\ldots$ & $\ldots$ & $\ldots$ & $\ldots$ & $\ldots$ & $0.00(0.00)$ & $3.57(0.26)$ & $4.13(0.34)$ & $3.84(0.31)$ & $2.29(0.26)$ & $1.18(0.16)$ & $>4.3$ \\
\hline $7430+1115$ & $\ldots$ & $\ldots$ & $\ldots$ & $\ldots$ & $\ldots$ & $\ldots$ & $0.99(0.02)$ & $4.83(0.28)$ & $5.79(0.34)$ & $2.71(0.33)$ & $1.42(0.26)$ & $0.39(0.02)$ & $>4.2$ \\
\hline $9477+2401^{d}$ & $\ldots$ & $\ldots$ & $\ldots$ & $\ldots$ & $\ldots$ & $\ldots$ & $0.15(0.03)$ & $0.57(0.04)$ & $0.53(0.04)$ & $0.69(0.03)$ & $0.26(0.03)$ & $0.00(0.00)$ & $>1.0$ \\
\hline $2223+4327$ & $0.29(0.01)$ & $0.54(0.02)$ & $1.79(0.42)$ & $0.63(0.02)$ & $0.55(0.02)$ & $0.17(0.01)$ & $0.00(0.00)$ & $0.63(0.04)$ & $1.08(0.33)$ & $0.70(0.03)$ & $0.75(0.03)$ & $0.21(0.01)$ & 1.3 \\
\hline $2574+6609$ & $\ldots$ & $\ldots$ & $\ldots$ & $\ldots$ & $\ldots$ & $\ldots$ & $0.00(0.00)$ & $5.55(0.26)$ & $2.71(0.34)$ & $3.68(0.33)$ & $1.10(0.27)$ & $0.19(0.02)$ & $>2.2$ \\
\hline $3304+6147$ & $\ldots$ & & $\ldots$ & $\ldots$ & $\ldots$ & $\ldots$ & $0.00(0.00)$ & $2.64(0.15)$ & $9.49(0.32)$ & $6.87(0.32)$ & $4.31(0.26)$ & $2.12(0.14)$ & $>2.6$ \\
\hline
\end{tabular}

In unit of $10^{-14} \mathrm{~W} / \mathrm{m}^{2}$. The numbers in brackets are fitting uncertainties.

no feature strengths derived for the 7 objects without SL observations.

The flux fractions are the ratios of the sum of all the AIB features to the total IR emission, which includes the contribution from the dust features from 10-35 $\mu \mathrm{m}$ and the dust thermal tinuum.

The flux measurements of this object may not be reliable (see section 2). 
Table 3. Peak wavelengths of AIB features. ${ }^{a}$

\begin{tabular}{ccccc}
\hline \hline IRAS ID & $6.2 \mu \mathrm{m}$ & $7.7-8.2 \mu \mathrm{m}$ & $8.6 \mu \mathrm{m}$ & $11.3 \mu \mathrm{m}$ \\
& & & & \\
\hline $01005+7910$ & $6.20 \pm 0.03$ & $7.57 \pm 0.05$ & $8.56 \pm 0.04$ & $11.25 \pm 0.02$ \\
$04296+3429$ & $6.26 \pm 0.02$ & $8.02 \pm 0.08$ & $\ldots$ & $11.42 \pm 0.09$ \\
$05113+1347$ & $\ldots$ & $\ldots$ & $\ldots$ & $11.41 \pm 0.13$ \\
$05341+0852$ & $\ldots$ & $\ldots$ & $\ldots$ & $11.40 \pm 0.09$ \\
$06530-0213$ & $\ldots$ & $\ldots$ & $\ldots$ & $11.38 \pm 0.02$ \\
$07430+1115$ & $\ldots$ & $\ldots$ & $\ldots$ & $11.40 \pm 0.13$ \\
$19477+2401$ & $\ldots$ & $\ldots$ & $\ldots$ & $11.38 \pm 0.13$ \\
$22223+4327$ & $6.29 \pm 0.03$ & $7.87 \pm 0.04$ & $8.53 \pm 0.03$ & $>11.30$ \\
$22574+6609$ & $\ldots$ & $\ldots$ & $\ldots$ & $11.31 \pm 0.04$ \\
$23304+6147$ & $\ldots$ & $\ldots$ & $\ldots$ & $11.40 \pm 0.06$ \\
\hline
\end{tabular}

${ }^{\mathrm{a}}$ In unit of $\mu \mathrm{m}$.

Table $4 . \mathrm{H}_{2}$ lines in IRAS $04296+3429$ and IRAS $22223+4327$.

\begin{tabular}{cccc}
\hline \hline IRAS ID & $\begin{array}{c}F(0-0 \mathrm{~S}(0)) \\
\left(\mathrm{W} / \mathrm{m}^{2}\right)\end{array}$ & $\begin{array}{c}F(0-0 \mathrm{~S}(1)) \\
\left(\mathrm{W} / \mathrm{m}^{2}\right)\end{array}$ & $\begin{array}{c}T_{\mathrm{ex}} \\
(\mathrm{K})\end{array}$ \\
\hline $04296+3429$ & $3.2 \mathrm{E}-14$ & $<4 \mathrm{E}-16$ & $<56$ \\
$22223+4327$ & $3.9 \mathrm{E}-14$ & $<2 \mathrm{E}-16$ & $<51$ \\
\hline
\end{tabular}

